\title{
Second-Kind Boundary Integral Equations for Scattering at Composite Partly Impenetrable Objects
}

\author{
Xavier Claeys ${ }^{1}$, Ralf Hiptmair ${ }^{2, *}$ and Elke Spindler ${ }^{2}$ \\ 1 Sorbonne Universités, UPMC Univ Paris 06, CNRS, INRIA, UMR 7598, \\ Laboratoire Jacques-Louis Lions, équipe Alpines, F-75005, Paris, France. \\ 2 Seminar for Applied Mathematics, Swiss Federal Institute of Technology, \\ $\mathrm{CH}-8092$ Zurich, Switzerland.
}

Received 4 October 2016; Accepted (in revised version) 4 May 2017

\begin{abstract}
We consider acoustic scattering of time-harmonic waves at objects composed of several homogeneous parts. Some of those may be impenetrable, giving rise to Dirichlet boundary conditions on their surfaces. We start from the recent secondkind boundary integral approach of [X. Claeys, and R. Hiptmair, and E. Spindler. A second-kind Galerkin boundary element method for scattering at composite objects. BIT Numerical Mathematics, 55(1):33-57, 2015] for pure transmission problems and extend it to settings with essential boundary conditions. Based on so-called global multipotentials, we derive variational second-kind boundary integral equations posed in $L^{2}(\Sigma)$, where $\Sigma$ denotes the union of material interfaces. To suppress spurious resonances, we introduce a combined-field version (CFIE) of our new method.

Thorough numerical tests highlight the low and mesh-independent condition numbers of Galerkin matrices obtained with discontinuous piecewise polynomial boundary element spaces. They also confirm competitive accuracy of the numerical solution in comparison with the widely used first-kind single-trace approach.
\end{abstract}

AMS subject classifications: $65 \mathrm{~N} 12,65 \mathrm{~N} 38,65 \mathrm{R} 20$

Key words: Acoustic scattering, second-kind boundary integral equations, Galerkin boundary element methods.

\section{Introduction}

\subsection{Acoustic scattering boundary value problem}

The governing equation for acoustic scattering of time-harmonic waves is the Helmholtz equation. In this article, we confine ourselves to the case of a globally constant principal part given by $-\Delta$.

*Corresponding author. Email addresses: xavier.claeys@upmc.fr (X. Claeys), hiptmair@sam.math.ethz.ch (R. Hiptmair), elke.spindler@sam.math.ethz.ch (E. Spindler) 


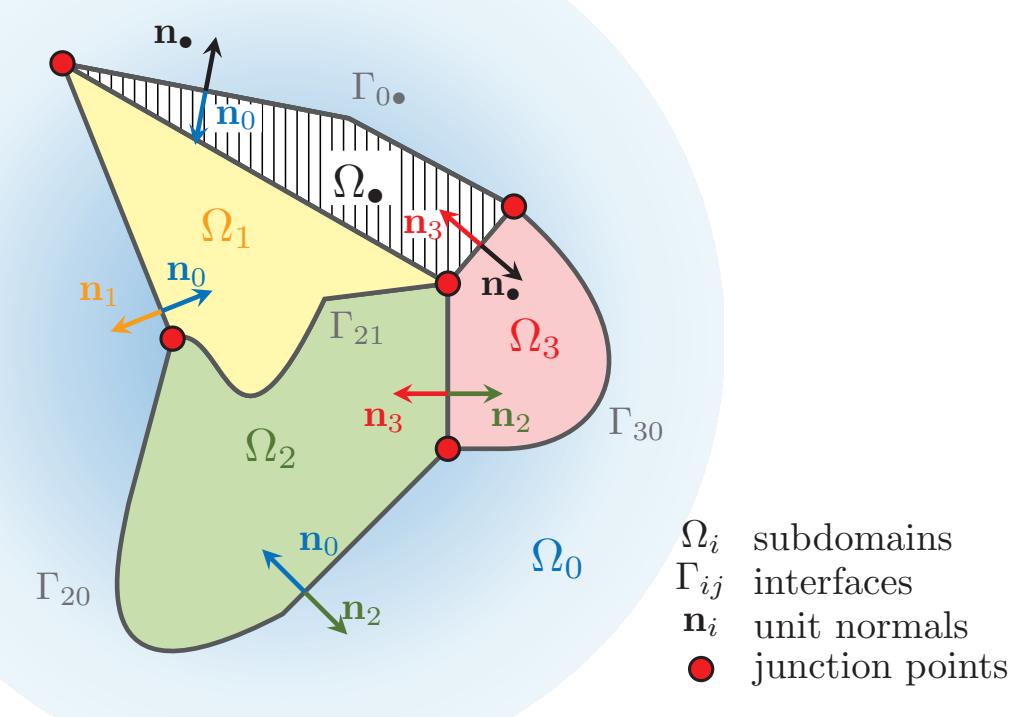

Figure 1: Two-dimensional illustration of a typical geometry of a composite scatterer for $L=3$.

The scatterer occupies a bounded domain $\Omega_{*} \subset \mathbb{R}^{d}, d=2,3$. We assume a partitioning of $\Omega_{*}$ into open Lipschitz subdomains, i.e. $\bar{\Omega}_{*}=\left(\bigcup_{i=1}^{L} \bar{\Omega}_{i}\right) \cup \bar{\Omega}_{\bullet}$, where $\bar{\Omega}$ denotes the closure of the domain $\Omega$. The subdomains $\Omega_{1}, \cdots, \Omega_{L}$ represent the different homogeneous penetrable materials whereas the impenetrable object with Lipschitz curvilinear polygonal/polyhedral boundary is given by $\Omega$. See Fig. 1 for a drawing of the scatterer in the case $d=2$. The unbounded exterior complement of the scatterer is given by the Lipschitz domain $\Omega_{0}:=\mathbb{R}^{d} \backslash \bar{\Omega}_{*}$. Like $\Omega_{1}, \cdots, \Omega_{L}$, also $\Omega_{0}$ is filled with homogeneous penetrable material. We characterize the penetrable materials by their wave numbers $\kappa_{i} \in \mathbb{R}_{+}$, for $i \in\{0,1, \cdots, L\}$. They enter the piecewise constant coefficient function $\kappa \in L^{\infty}\left(\mathbb{R}^{d}\right),\left.\kappa\right|_{\Omega_{i}} \equiv \kappa_{i}$. The impenetrable object $\Omega$. will be modeled by imposing Dirichlet boundary conditions at its boundary $\partial \Omega$.

By construction, we observe that $\Omega_{i} \cap \Omega_{j}=\varnothing$ for $j \neq i$, for indices $i, j \in\{\bullet, 0,1, \cdots, L\}$. The boundary of the subdomain $\Omega_{i}$ is given by $\partial \Omega_{i}$ for $i \in\{\bullet, 0,1 \cdots, L\}$. For Lipschitz domains, and in particular for each $\Omega_{i}$, there exists a unit normal vector field $\mathbf{n}_{i} \in L^{\infty}\left(\partial \Omega_{i}\right)$, $\mathbf{n}_{i}: \partial \Omega_{i} \rightarrow \mathbb{R}^{d}$, pointing towards the exterior of $\Omega_{i}$.

The interface between two subdomains $\Omega_{i}$ and $\Omega_{j}$ is denoted by $\Gamma_{i j}:=\partial \Omega_{i} \cap \partial \Omega_{j}$. Moreover, we introduce the so-called skeleton $\Sigma:=\bigcup_{i=0}^{L} \partial \Omega_{i}$, the union of all boundaries of subdomains.

In our scattering model sources are given through an incident wave, coming from infinity and impinging on the scattering obstacle. We assume that the source field $U_{\text {inc }} \in$ 
$C^{\infty}\left(\mathbb{R}^{d}\right)^{\dagger}$ satisfies the Helmholtz equation

$$
-\Delta U_{\text {inc }}-\kappa_{0}^{2} U_{\text {inc }}=0 \quad \text { everywhere in } \mathbb{R}^{d},
$$

where $\kappa_{0}$ denotes the wave number corresponding to the exterior unbounded domain $\Omega_{0}$.

Now we are in a position to state the acoustic scattering problem in variational form: Seek $U \in H_{0, \text { loc }}^{1}\left(\mathbb{R}^{d} \backslash \bar{\Omega}_{\bullet}\right)$ such that

$$
\int_{\mathbb{R}^{d} \backslash \bar{\Omega}} \operatorname{grad} U(\boldsymbol{x}) \cdot \operatorname{grad} V(\boldsymbol{x})-\kappa^{2}(\boldsymbol{x}) U(\boldsymbol{x}) V(\boldsymbol{x}) \mathrm{d} \boldsymbol{x}=0,
$$

for all $V \in H_{0, \text { comp }}^{1}\left(\mathbb{R}^{d} \backslash \bar{\Omega}_{\bullet}\right)$, and the scattered field $U_{s}:=U-U_{\text {inc }}$ satisfies the Sommerfeld radiation condition [14, Sect. 2.2]

$$
\lim _{r \rightarrow \infty} \int_{|x|=r}\left|\operatorname{grad} U_{s}(\boldsymbol{x}) \cdot \frac{\boldsymbol{x}}{|\boldsymbol{x}|}-\mathrm{i} \kappa_{0} U_{s}(\boldsymbol{x})\right|^{2} \mathrm{~d} S(\boldsymbol{x})=0 .
$$

Existence and uniqueness of solutions to (1.2) are well established [38, Sect. 2]. ${ }^{\ddagger}$

Remark 1.1 (Transmission Conditions). The variational formulation (1.2a) implies socalled transmission conditions

$$
\left.U\right|_{\partial \Omega_{i}}=\left.U\right|_{\partial \Omega_{j}{ }^{\prime}} \quad \mathbf{n}_{i} \cdot\left[\left.(\operatorname{grad} U)\right|_{\partial \Omega_{i}}\right]=-\mathbf{n}_{j} \cdot\left[\left.(\operatorname{grad} U)\right|_{\partial \Omega_{j}}\right],
$$

which hold on $\Gamma_{i j}=\partial \Omega_{i} \cap \partial \Omega_{j}, i, j \in\{\bullet, 0, \ldots, L\}, i \neq j$, in the sense of distributions.

Remark 1.2 (Piecewise constant diffusion coefficient). Instead of (1.2a) we may consider

$$
\int_{\mathbb{R}^{d} \backslash \bar{\Omega}_{\bullet}} \alpha(\boldsymbol{x}) \operatorname{grad} U(\boldsymbol{x}) \cdot \operatorname{grad} V(\boldsymbol{x})-\kappa^{2}(\boldsymbol{x}) U(\boldsymbol{x}) V(\boldsymbol{x}) \mathrm{d} \boldsymbol{x}=0,
$$

with a piecewise constant positive diffusion coefficient function $\alpha \in L^{\infty}\left(\mathbb{R}^{d}\right)$. Our approach can be extended to this setting by switching to a scaled multi-potential generalizing (2.9), as has been done in [13]. In the context of electromagnetic scattering, this scaling trick is discussed in [11], see Definition 4.1 in that manuscript. It will achieve regularization by cancellation of kernel singularities of the hypersingular boundary integral operator as in (4.8). Yet, for the double layer boundary integral operators this cancellation will no longer occur. Nevertheless, the resulting Cauchy-singular skeleton boundary integral operators will still be continuous in $L^{2}$ skeleton spaces and yield a valid second kind formulation. In this article we forgo this generalization for the sake of simplicity and because an implementation for acoustic scattering is not yet available to us.

\footnotetext{
${ }^{\dagger}$ Capital letters are used to refer to functions defined over a volume domain.

$\ddagger$ Notations for function spaces (Sobolev spaces) follow the usual conventions, see $[9,28]$. In particular, we write $H_{\text {loc }}^{s}(\Omega)$ for distributions $U$ such that $\left.\phi\right|_{\Omega} U \in H^{s}(\Omega)$ for any $\phi \in C_{\text {comp }}^{\infty}\left(\mathbb{R}^{d}\right)$, see [35, Definition 2.6.1]. $H_{\text {comp }}^{s}(\Omega)$ contains all distributions in $H_{\text {loc }}^{s}(\Omega)$ that have compact support in $\Omega$, see [35, Definition 2.6.5] and $H_{0, \mathrm{loc}}^{1}(\Omega)$ consists of all distributions in $H_{\mathrm{loc}}^{s}(\Omega)$ that vanish on $\partial \Omega$.
} 


\subsection{Second-kind boundary integral formulations}

Boundary integral equations (BIE) are an effective tool to model the acoustic scattering of waves at partly impenetrable objects consisting of several homogeneous materials. They form the foundation for Galerkin boundary element methods (BEM), a popular class of methods to discretize and numerically compute acoustic fields. BEM are - in contrast to finite element methods - well-suited for scattering problems, since they can easily deal with unbounded domains. Second-kind BIE for transmission problems in the case of a homogeneous scatterer are well established, that is, when there is no impenetrable object $\Omega$. and $L=1$ (see [27,30,34] or [14, Sect. 3] or [35, Sect. 3.9]). As well understood are second-kind BIE for exterior Dirichlet and Neumann problems (c.f. [14, Sect. 3] or [35, Sect. 3.9], [16, 17]), i.e. the case described in Subsection 1.1 for $L=0$. In this case, the occurrence of spurious resonances is a persistent problem and combined field integral equations (CFIE) are a popular remedy, see [3,4].

Our aim is to unify these approaches to treat complex scatterers consisting of impenetrable as well as several penetrable homogeneous materials.

\subsection{First-kind boundary integral formulations}

For the geometric situation described in Subsection 1.1, a widely used BIE is the first-kind single-trace formulation (STF) [15, 38], in computational electromagnetics also known as Poggio-Miller-Chang-Harrington-Wu-Tsai (PMCHWT) integral equation [5, 21,33,39]. Other recently developed approaches to solve the same type of problems are various kinds of multi-trace formulations (MTF), see [7-10, 24, 31,32]. The boundary element Galerkin discretization of the classical first-kind STF as well as of the MTF leads to illconditioned linear systems on fine meshes. Therefore iterative solvers require preconditioning, which means additional coding and computational effort.

\subsection{Novelty and outline}

In this article, we extend the Galerkin BEM approach for second-kind STF for transmission problems proposed in [12] to partly impenetrable objects. This type of STF is based on so-called multi-potentials (see Subsection 2.2). we find that it is possible to consider the (variational) second-kind STF in $L^{2}$. Thus, a boundary element Galerkin discretization based on the new formulations yields intrinsically well-conditioned linear systems. To overcome spurious resonances, we adopt the idea of (direct) combined field integral equations (CFIE, see [3,4]) in Section 3. The treatment of essential boundary conditions along with a CFIE-type stabilization of second-kind skeleton BIEs are major innovations in this work. They deserve attention, because numerical tests in three dimensions provide solid evidence for

(i) stability of our second-kind Galerkin discretization,

(ii) mesh-independent good conditioning of the Galerkin matrices, and 
(iii) competitive accuracy in comparison with the first-kind STF.

We emphasize that the focus of the present work is not on theoretical investigations, but on the derivation of the new boundary integral equations, the implementation of the related Galerkin BEM, and numerical tests probing specific properties in typical settings. Theoretical considerations will only be recalled briefly and for proofs we largely refer to $[6,12,36]$.

In Section 2 we first present the basic tools, set up the notation, and introduce the spaces needed for our second-kind single-trace formulation. There we also introduce the formulation itself and, afterwards in Section 3, we derive its CFIE extension. Based on a specific example, the discretization and implementation of the method is discussed in Section 4. Finally, we report on numerical results in Section 5.

\section{Boundary integral equations}

\subsection{Traces and potentials $\ddagger$}

For the $i$ th subdomain, $i \in\{\bullet, *, 0,1, \cdots, L\}$, we introduce the interior Dirichlet and Neumann trace (co-normal trace) [35, Theorems 2.6.9, 2.8.3 \& Lemma 2.8.4 $]^{\S}$

$$
\begin{array}{ll}
\gamma_{D}^{i}: H_{\mathrm{loc}}^{1}\left(\Omega_{i}\right) \rightarrow H^{\frac{1}{2}}\left(\partial \Omega_{i}\right), & \gamma_{D}^{i} u:=u_{\mid \partial \Omega_{i}}, \\
\gamma_{N}^{i}: H_{\mathrm{loc}}^{1}\left(\Delta, \Omega_{i}\right) \rightarrow H^{-\frac{1}{2}}\left(\partial \Omega_{i}\right), & \gamma_{N}^{i} u:=\mathbf{n}_{i} \cdot \operatorname{grad} u_{\mid \partial \Omega_{i}} .
\end{array}
$$

The exterior Dirichlet and Neumann traces $\gamma_{D}^{i, c}: H_{\text {loc }}^{1}\left(\mathbb{R}^{d} \backslash \bar{\Omega}_{i}\right) \rightarrow H^{\frac{1}{2}}\left(\partial \Omega_{i}\right)$ and $\gamma_{N}^{i, c}$ : $H_{\text {loc }}^{1}\left(\Delta, \mathbb{R}^{d} \backslash \bar{\Omega}_{i}\right) \rightarrow H^{-\frac{1}{2}}\left(\partial \Omega_{i}\right)$, are taken from outside $\Omega_{i}$. The associated trace spaces, henceforth called Dirichlet trace space and Neumann trace space, can be merged into the Cauchy trace space $\mathcal{H}\left(\partial \Omega_{i}\right):=H^{\frac{1}{2}}\left(\partial \Omega_{i}\right) \times H^{-\frac{1}{2}}\left(\partial \Omega_{i}\right)$, which is self-dual with respect to the pairing

$$
\langle\langle\mathfrak{u}, \mathfrak{v}\rangle\rangle_{\mathcal{H}\left(\partial \Omega_{i}\right)}:=\langle u, \varphi\rangle_{\partial \Omega_{i}}-\langle v, v\rangle_{\partial \Omega_{i},} \quad \mathfrak{u}:=\left(\begin{array}{c}
u \\
v
\end{array}\right), \quad \mathfrak{v}:=\left(\begin{array}{c}
v \\
\varphi
\end{array}\right) \in \mathcal{H}\left(\partial \Omega_{i}\right),
$$

with $\langle\cdot, \cdot\rangle_{\partial \Omega_{i}}$ denoting (extensions of) the $L^{2}$-duality pairing on $\partial \Omega_{i}$. A related compact notation is the Cauchy trace operator

$$
\gamma^{i}: H_{\mathrm{loc}}^{1}\left(\Delta, \Omega_{i}\right) \rightarrow \mathcal{H}\left(\partial \Omega_{i}\right), \quad \gamma^{i} U:=\left(\begin{array}{c}
\gamma_{D}^{i} U \\
\gamma_{N}^{i} U
\end{array}\right)
$$

Potential representations of solutions of (1.2) are the first step towards boundary integral equations. The following result can be found in [35, Sect. 3.11] and [28, Ch. 6]:

$\ddagger$ We are going to use the same notation as introduced in [12, Subsection 3.1].

$\S_{H_{\text {loc }}^{1}}^{1}(\Delta, \Omega):=\left\{U \in H_{\text {loc }}^{1}(\Omega) \mid \Delta U \in L_{\text {comp }}^{2}(\Omega)\right\}$, see [35, Equation (2.108)]. 
Lemma 2.1 (Single Domain Representation Formula). There are continuous linear operators, depending on the constant $\kappa>0$, the

$$
\begin{aligned}
\text { single layer potential } & \mathbb{S}_{i}[\kappa]: H^{-\frac{1}{2}}\left(\partial \Omega_{i}\right) \rightarrow H_{\mathrm{loc}}^{1}\left(\Delta, \mathbb{R}^{d} \backslash \partial \Omega_{i}\right), \\
\text { double layer potential } & \mathbb{D}_{i}[\kappa]: H^{\frac{1}{2}}\left(\partial \Omega_{i}\right) \rightarrow H_{\mathrm{loc}}^{1}\left(\Delta, \mathbb{R}^{d} \backslash \partial \Omega_{i}\right),
\end{aligned}
$$

such that

(i) For any $\varphi \in H^{-\frac{1}{2}}\left(\partial \Omega_{i}\right), u \in H^{\frac{1}{2}}\left(\partial \Omega_{i}\right)$ the potentials $S_{i}[\kappa](\varphi)$ and $\mathbb{D}_{i}[\kappa](u)$ are solutions of $-\Delta U-\kappa^{2} U=0$ in $\Omega_{i}$ and in $\mathbb{R}^{d} \backslash \overline{\Omega_{i}}$ and satisfy the Sommerfeld radiation conditions $(1.2 b)$.

(ii) Every solution $U \in H_{\mathrm{loc}}^{1}\left(\Omega_{i}\right)$ of $\left(-\Delta-\kappa^{2}\right) U=0$ that satisfies the Sommerfeld radiation conditions $(1.2 \mathrm{~b})$ if $i=0$ fulfills

$$
\mathbb{G}_{i}[\kappa]\left(\gamma^{i} U\right)= \begin{cases}U & \text { on } \Omega_{i}, \\ 0 & \text { on } \mathbb{R}^{d} \backslash \overline{\Omega_{i}},\end{cases}
$$

with the local potentials defined by $\mathbb{I}$

$$
\mathbb{G}_{i}[\kappa](\mathfrak{u}):=-\mathbb{D}_{i}[\kappa](u)+\mathbb{S}_{i}[\kappa](\varphi), \quad \mathfrak{u}:=\left(\begin{array}{c}
u \\
\varphi
\end{array}\right) \in \mathcal{H}\left(\partial \Omega_{i}\right) .
$$

For distributions $\varphi$ and $u$ on $\partial \Omega_{i}$ the potentials possess the integral representations

$$
\begin{aligned}
& \mathrm{S}_{i}[\kappa](\varphi)(\boldsymbol{x})=\int_{\partial \Omega_{i}} \Phi_{\kappa}(\boldsymbol{x}-\boldsymbol{y}) \varphi(\boldsymbol{y}) \mathrm{d} S(\boldsymbol{y}), \\
& \mathbb{D}_{i}[\kappa](u)(\boldsymbol{x})=\int_{\partial \Omega_{i}} \operatorname{grad}_{\boldsymbol{y}} \Phi_{\kappa}(\boldsymbol{x}-\boldsymbol{y}) \cdot \mathbf{n}_{i}(\boldsymbol{y}) u(\boldsymbol{y}) \mathrm{d} S(\boldsymbol{y}),
\end{aligned}
$$

for $x \notin \partial \Omega_{i}$, based on fundamental solutions

$$
\Phi_{\kappa}(z)=\left\{\begin{array}{ll}
\frac{\mathrm{i}}{4} H_{0}^{(1)}(\kappa|z|), & d=2, \\
\frac{1}{4 \pi|z|} \exp (\mathrm{i} \kappa|z|), & d=3,
\end{array} \quad \kappa \in \mathbb{R}_{+},\right.
$$

where $H_{0}^{(1)}$ is the Hankel function of the first kind and $|\cdot|$ represents the Euclidean norm.

Notation. For simplicity we neglect the argument $[\kappa]$ in $S_{i}[\kappa], \mathbb{D}_{i}[\kappa]$, and $\mathbb{G}_{i}[\kappa]$ and write $S_{i}:=S_{i}\left[\kappa_{i}\right], \mathbb{D}_{i}:=\mathbb{D}_{i}\left[\kappa_{i}\right], \mathbb{G}_{i}:=\mathbb{G}_{i}\left[\kappa_{i}\right]$, respectively, in the cases where $\kappa$ in the formulas (2.4) coincides with the local wave number $\kappa_{i}$ of $\Omega_{i}, i \in\{0,1, \cdots, L\}$.

IIFraktur font is used to designate functions in the Cauchy trace space, where Roman typeface is reserved for Dirichlet traces, and Greek symbols for Neumann traces. 


\section{2 $L^{2}$ skeleton trace spaces and multi-potentials}

This subsection will follow the same notation as introduced in [12, Subsections 3.2,3.3] or [6, Section 2], but will extend it to the setting with impenetrable material in $\Omega_{\bullet}$. Moreover, for the sake of conciseness, we consider a functional setting based on square integrable functions rather than on Sobolev spaces of fractional order.

Definition 2.1 (Multi-Trace Space [6, Section 2], [12, Def. 3.1], [36, Def. 3.2.1]). The $L^{2}$ skeleton multi-trace space is given by

$$
\mathcal{M L}^{2}(\Sigma):=\prod_{i=0}^{L} L^{2}\left(\partial \Omega_{i}\right) \times L^{2}\left(\partial \Omega_{i}\right)
$$

Next for any $\epsilon>0$, we may introduce the skeleton multi-trace operator $\gamma^{\Sigma}$, mapping $H_{\text {loc }}^{3 / 2+\epsilon}\left(\mathbb{R}^{d} \backslash\left(\Omega_{\bullet} \cup \Sigma\right)\right)=H_{\text {loc }}^{3 / 2+\epsilon}\left(\Omega_{0}\right) \times \cdots \times H_{\text {loc }}^{3 / 2+\epsilon}\left(\Omega_{L}\right)$ into the multi-trace space, is given by $\gamma^{\Sigma}: H_{\text {loc }}^{3 / 2+\epsilon}\left(\mathbb{R}^{d} \backslash\left(\Omega_{\bullet} \cup \Sigma\right)\right) \rightarrow \mathcal{M L}^{2}(\Sigma)$,

$$
\gamma^{\Sigma} U:=\left(\gamma^{0} U, \gamma^{1} U, \cdots, \gamma^{L} U\right)
$$

We notice self-duality of $\mathcal{M} \mathcal{L}^{2}(\Sigma)$ with respect to an $L^{2}$-type bilinear pairing defined, for any $\mathfrak{u}=\left(\mathfrak{u}_{j}\right)_{j=0}^{L} \in \mathcal{M} \mathcal{L}^{2}(\Sigma)$ and $\mathfrak{v}=\left(\mathfrak{v}_{j}\right)_{j=0}^{L} \in \mathcal{M} \mathcal{L}^{2}(\Sigma)$, by the formula

$$
\begin{aligned}
\langle\langle\mathfrak{u}, \mathfrak{v}\rangle\rangle & :=\sum_{i=0}^{L}\left\langle\left\langle\mathfrak{u}_{i}, \mathfrak{v}_{i}\right\rangle\right\rangle_{\mathcal{H}\left(\partial \Omega_{i}\right)} \\
& =\sum_{0 \leq j<i \leq L_{\Gamma_{i j}}} \int_{i} u_{i} \varphi_{i}-v_{i} v_{i}+u_{j} \varphi_{j}-v_{j} v_{j} \mathrm{~d} S+\sum_{0 \leq i \leq L} \int_{\Gamma_{i} \bullet} u_{i} \varphi_{i}-v_{i} v_{i} \mathrm{~d} S,
\end{aligned}
$$

where $\mathfrak{u}_{i}=\left(u_{i}, v_{i}\right), \mathfrak{v}_{i}=\left(v_{i}, \varphi_{i}\right)$. Next, we introduce the important subspace of unique traces in $\mathcal{M L}^{2}(\Sigma)$ that incorporate homogeneous Dirichlet boundary conditions at $\partial \Omega$.

Definition 2.2 ( $L^{2}$ Single-Trace Space [12, Def. 4.2], [36, Def. 4.2.6]). The $L^{2}$ single-trace space is defined by

$$
\begin{aligned}
\mathcal{S} \mathcal{L}^{2}(\Sigma):=\{ & \left(u_{0}, v_{0}, \ldots, u_{L}, v_{L}\right) \in \mathcal{M} \mathcal{L}^{2}(\Sigma):\left.\quad u_{i}\right|_{\Gamma_{i j}}=\left.u_{j}\right|_{\Gamma_{i j}{ }^{\prime}} \\
& \left.\left.v_{i}\right|_{\Gamma_{i j}}=-\left.v_{j}\right|_{\Gamma_{i j}}, \forall j<i \text { and }\left.u_{i}\right|_{\partial \Omega .}=0, \forall i \in\{0, \cdots, L\}\right\} .
\end{aligned}
$$

Elements of $\mathcal{S T}(\Sigma)$ should be regarded as skeleton traces of functions defined everywhere on $\mathbb{R}^{d} \backslash \bar{\Omega}_{\bullet}$. Indeed for any $\epsilon>0$, an element $\left(u_{j}, v_{j}\right)_{j=0}^{L} \in \mathcal{M L}^{2}(\Sigma)$ such that $u_{j} \in H^{\epsilon}\left(\partial \Omega_{j}\right)$ actually belongs to $\mathcal{S} \mathcal{L}^{2}(\Sigma)$ if and only if there exist two globally defined volume based functions $U \in H_{0}^{\epsilon+1 / 2}\left(\mathbb{R}^{d} \backslash \bar{\Omega}_{\bullet}\right)$ and $\boldsymbol{\phi} \in H\left(\operatorname{div}, \mathbb{R}^{d} \backslash \bar{\Omega}_{\bullet}\right)$ such that $\gamma_{D}^{i}(U)=u_{j}$ and $\mathbf{n}_{i} \cdot\left(\gamma_{D}^{i}, \gamma_{D}^{i}\right) \boldsymbol{\phi}=v_{i}$. 
Even in an $L^{2}$ setting we can still capture the traces of solutions of the scattering transmission problem (1.2), because, appealing to elliptic lifting results [26, Theorem B.2], [20, Remark 2.4.6, Corollary 2.6.7], we observe that the solution $U$ of (1.2) belongs to $H_{\text {loc }}^{\frac{3}{2}+\epsilon}\left(\mathbb{R}^{d} \backslash \bar{\Omega}_{\bullet}\right)$ for some $\epsilon>0$. Then, standard trace theorems supply the following regularity even of Neumann traces of $U$ :

Lemma 2.2. For the unique solution $U$ of (1.2) holds $\gamma^{\Sigma} U \in \mathcal{S L}^{2}(\Sigma)$.

The polar set characterization of $\mathcal{S L}^{2}(\Sigma)$ as a subspace of $\mathcal{M L}^{2}(\Sigma)$, see also [6, Prop. 2.1], [9, Thm. 3.1] and [12], still holds for our extension to impenetrable parts.

Lemma 2.3.

$$
\mathcal{S L}^{2}(\Sigma)=\left\{\mathfrak{u} \in \mathcal{M L}^{2}(\Sigma):\langle\langle\mathfrak{u}, \mathfrak{v}\rangle\rangle=0 \forall \mathfrak{v} \in \mathcal{S L}^{2}(\Sigma)\right\} .
$$

Based on the spaces introduced above, we define the so-called multi-potential.

Lemma 2.4 ([36, Def. 3.3.7], [36, Thm. 3.3.13, Lem. 4.3.4, Lem. 4.3.6]). The multi-potential is defined as the sum of all local potentials $\mathbb{G}_{i}\left[\kappa_{i}\right]$ defined in Lemma $2.1, i=0, \cdots, L$ :

$$
\mathbb{M}_{\Sigma}(\mathfrak{u}):=\sum_{i=0}^{L} \mathrm{G}_{i}\left[\kappa_{i}\right]\left(\mathfrak{u}_{i}\right)
$$

Moreover the boundary integral operator obtained by taking its traces $\mathbf{M}_{\Sigma}:=\gamma^{\Sigma} \mathbf{M}_{\Sigma}$ induces a continuous linear map $\mathcal{M L}^{2}(\Sigma) \rightarrow \mathcal{M L}^{2}(\Sigma)$.

Notation. If $\mathbb{M}_{\Sigma}$ or $\mathbf{M}_{\Sigma}$ are supplied with an argument [ $\left.\kappa\right]$, all wave numbers $\kappa_{i}$ in (2.9) are supposed to agree with $\kappa$ in $\mathbb{M}_{\Sigma}[\kappa]$ and $\mathbf{M}_{\Sigma}[\kappa]$, respectively.

The attribute multi indicates that generically $L+1$ potentials contribute to the value of $\mathbb{M}_{\Sigma}(\mathfrak{u})(x)$ for every $x \notin \Sigma$. Yet, the multi-potential becomes single-valued when acting on traces of the solution of (1.2). To see this note that, if $U$ solves the transmission problem (1.2), then

$$
\begin{aligned}
& \mathbb{G}_{i}\left[\kappa_{i}\right]\left(\gamma^{i} U\right)=\left\{\begin{array}{ll}
U & \text { in } \Omega_{i}, \\
0 & \text { elsewhere, }
\end{array} \quad i=1, \cdots, L,\right. \\
& \mathbb{G}_{0}\left[\kappa_{0}\right]\left(\gamma^{0}\left(U-U_{\text {inc }}\right)\right)= \begin{cases}U-U_{\text {inc }} & \text { in } \Omega_{0}, \\
0 & \text { elsewhere. }\end{cases}
\end{aligned}
$$

This explains the following multi-potential representation formula.

Corollary 2.1 (Global Representation Formula [12, Cor. 3.1], [36, Cor. 3.3.8]). If U solves the transmission problem (1.2), then

$$
U-\left.U_{\mathrm{inc}}\right|_{\Omega_{0}}=\mathbb{M}_{\Sigma} \gamma^{\Sigma}\left(U-\left.U_{\mathrm{inc}}\right|_{\Omega_{0}}\right),
$$

where $\gamma^{\Sigma}$ is the multi-trace defined in (2.7). 
The representation formula in (2.10) paves the way to the boundary integral formulation. We take the skeleton-trace on both sides of the equation, insert the resulting equation into the bilinear form (2.8) and test with $\mathfrak{v} \in \mathcal{M} \mathcal{T}(\Sigma)$. We obtain the following variational BIE satisfied by $\mathfrak{u}:=\gamma^{\Sigma} U$.

Formulation 2.1 ([36, Form. 4.3.3]). Find $\mathfrak{u} \in \mathcal{S L}^{2}(\Sigma)$ such that

$$
\left\langle\left\langle\left(\mathbf{I d}-\mathbf{M}_{\Sigma}\right) \mathfrak{u}, \mathfrak{v}\right\rangle\right\rangle=\left\langle\left\langle\mathfrak{u}_{\text {inc }}, \mathfrak{v}\right\rangle\right\rangle \quad \forall \mathfrak{v} \in \mathcal{M L}^{2}(\Sigma), \quad \mathfrak{u}_{\text {inc }}:=\gamma^{\Sigma} U_{\text {inc }} .
$$

The simple expression on the right hand side is due to the identity

$$
\left\langle\left\langle\left(\mathbf{I d}-\mathbf{M}_{\Sigma}\right) \mathfrak{u}_{\text {inc }}^{0}, \mathfrak{v}\right\rangle\right\rangle=\left\langle\left\langle\mathfrak{u}_{\text {inc }}, \mathfrak{v}\right\rangle\right\rangle .
$$

It holds, since we assume that the incident wave $U_{\text {inc }}$ solves an interior Helmholtz problem on $\Omega_{*}$, see (1.1). To be more precise, by (2.3), we obtain

$$
\mathbb{G}_{*}\left[\mathcal{K}_{0}\right]\left(\gamma^{*} U_{\text {inc }}\right)= \begin{cases}U_{\text {inc }} & \text { on } \Omega_{*}, \\ 0 & \text { on } \mathbb{R}^{d} \backslash \bar{\Omega}_{*} .\end{cases}
$$

Since $\Omega_{0}=\mathbb{R}^{d} \backslash \bar{\Omega}_{*}$, this involves

$$
\mathrm{G}_{*}\left[\kappa_{0}\right]\left(\gamma^{*} U_{\text {inc }}\right)+\left.U_{\text {inc }}\right|_{\Omega_{0}}=U_{\text {inc }} .
$$

Taking into account the relation between $\mathbb{G}_{*}$ and $\mathbb{G}_{0}$ together with the fact that

$$
\gamma^{*} U_{\text {inc }}=\left(\begin{array}{c}
\gamma_{D}^{0} \\
-\gamma_{N}^{0}
\end{array}\right) U_{\text {inc }}
$$

we can write (2.11) in terms of the multi potential (2.9). We observe

$$
-\mathbb{M}_{\Sigma}\left(\gamma^{\Sigma}\left(\left.U_{\text {inc }}\right|_{\Omega_{0}}\right)\right)+\left.U_{\text {inc }}\right|_{\Omega_{0}}=U_{\text {inc }}
$$

which finally yields

$$
\left(\mathbf{I d}-\mathbf{M}_{\Sigma}\right) \mathfrak{u}_{\text {inc }}^{0}=\gamma^{\Sigma} U_{\text {inc }}=\mathfrak{u}_{\text {inc }} .
$$

We observe an important redundancy in the variational form of the boundary integral equations in Formulation 2.1:

$$
\left\langle\left\langle\left(\mathrm{Id}-\mathbf{M}_{\Sigma}\right) \gamma^{\Sigma} U, \mathfrak{v}\right\rangle\right\rangle=0, \quad \forall \mathfrak{v} \in \mathcal{S} \mathcal{L}^{2}(\Sigma),
$$

if $U$ is the solution of (1.2). In words, testing with single-trace functions yields " $0=0$ " and does not supply any information. The relationship (2.12) not only points to redundancy in the test space of Formulation 2.1, it also hints at a remedy. Since $\mathcal{S L}^{2}(\Sigma)$ is a closed subspace of $\mathcal{M L}^{2}(\Sigma)$ (as an immediate corollary of Lemma 2.3), it is sufficient to test with elements in the $L^{2}$-orthogonal complement of $\mathcal{S L}^{2}(\Sigma)$ in $\mathcal{M L}^{2}(\Sigma)$, which enjoys a simple characterization, see [36, Def. 4.3.11]. 
Definition 2.3 (Orthogonal Complement of the Single-Trace $L^{2}$-Space).

$$
\begin{aligned}
\mathcal{S L}^{2, \perp}(\Sigma):=\{ & \left(u_{0}, v_{0}, \cdots, u_{L}, v_{L}\right) \in \mathcal{M} \mathcal{L}^{2}(\Sigma):\left.u_{i}\right|_{\Gamma_{i j}}=-\left.u_{j}\right|_{\Gamma_{i j}{ }^{\prime}} \\
& \left.\left.v_{i}\right|_{\Gamma_{i j}}=\left.v_{j}\right|_{\Gamma_{i j}{ }^{\prime}} j<i \text { and }\left.v_{i}\right|_{\Gamma_{i \bullet}}=0 \forall i \in\{0,1, \cdots, L\}\right\} .
\end{aligned}
$$

Testing with functions in this complement space leads to the following variational BIE from Formulation 2.1.

Formulation 2.2 ([36, Form. 4.3.12]). Search $\mathfrak{u} \in \mathcal{S L}^{2}(\Sigma)$ :

$$
\left\langle\left\langle\left(\mathrm{Id}-\mathbf{M}_{\Sigma}\right) \mathfrak{u}, \mathfrak{v}\right\rangle\right\rangle=\left\langle\left\langle\mathfrak{u}_{\text {inc }}, \mathfrak{v}\right\rangle\right\rangle, \quad \forall \mathfrak{v} \in \mathcal{S} \mathcal{L}^{2, \perp}(\Sigma), \quad \mathfrak{u}_{\text {inc }}:=\gamma^{\Sigma} U_{\text {inc }} .
$$

Corollary 2.2. Formulation 2.2 is consistent with the original scattering problem in the sense that the exact solution of (1.2) will also fulfill Formulation 2.2.

As a consequence of $\gamma^{\Sigma} U \in \mathcal{S} \mathcal{L}^{2}(\Sigma)$ we can state the equivalence of Formulation 2.2 to the original problem (1.2).

Corollary 2.3 (Equivalence [36, Cor. 4.3.16]). If Formulation 2.2 has a unique solution $\mathfrak{u} \in$ $\mathcal{S L}^{2}(\Sigma)$, then $\mathfrak{u}$ provides the skeleton trace $\gamma^{\Sigma} U$ of the solution $U$ of the original transmission problem (1.2).

The proof of Corollary 2.3 runs parallel to that of [12, Corollary 4.2].

Remark 2.1. [12, Thm. 4.1] asserts that the operator underlying Formulation 2.2 is a compact perturbation of the identity when there is no impenetrable material. In this case the proof of well-posedness of Formulation 2.2 reduces to the verification of

$$
\operatorname{Ker}\left(\mathrm{Id}-\mathbf{M}_{\Sigma}\right)=\{0\}
$$

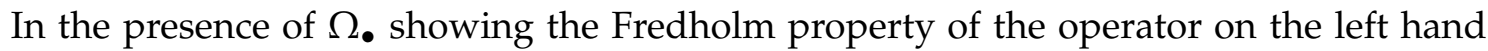
side of Formulation 2.2 is much more involved and could only be accomplished for $d=2$ under additional geometric assumptions, see [36, Appendix C].

It is still open, whether (2.13) holds true. But numerical tests (see [12, Subsection 6.1]) indicate the absence of spurious resonances in the absence of impenetrable materials. Otherwise, spurious resonances are known to affect the formulation for particular wave numbers. A heuristic remedy will be devised in Section 3.

\subsection{Formulation in $L^{2}$ interface trace spaces}

Efficient implementation must be interface-oriented and, therefore, we now look at the $L^{2}$ skeleton trace spaces from a different perspective. 
Definition 2.4 ( $L^{2}$ Skeleton Trace Space, [36, eq. (4.9)]). The $L^{2}$ skeleton trace space is given by

$$
\mathcal{L}_{\bullet}^{2}(\Sigma):=\left(\prod_{0 \leq j<i \leq L} L^{2}\left(\Gamma_{i j}\right) \times L^{2}\left(\Gamma_{i j}\right)\right) \times\left(\prod_{0 \leq i \leq L}\{0\} \times L^{2}\left(\Gamma_{i \bullet}\right)\right) .
$$

In order to make sense of the notation $\mathfrak{u}=\left(u_{i j}, v_{i j}\right)_{j<i} \in \mathcal{L}_{\bullet}^{2}(\Sigma)$, we set $\bullet<j$ for all $j \in$ $\{0, \cdots, L\}$. It is a closed subspace of the space

$$
\mathcal{L}^{2}(\Sigma):=\left(\prod_{0 \leq j<i \leq L} L^{2}\left(\Gamma_{i j}\right) \times L^{2}\left(\Gamma_{i j}\right)\right) \times\left(\prod_{0 \leq i \leq L} L^{2}\left(\Gamma_{i \bullet}\right) \times L^{2}\left(\Gamma_{i \bullet}\right)\right),
$$

also allowing for non-vanishing Dirichlet contributions at the boundary of the impenetrable subdomain $\partial \Omega$.

It is isomorphic to $\mathcal{S} \mathcal{L}^{2}(\Sigma)$ by the following one-to-one correspondence. Any element $\mathfrak{u}=\left(u_{i j}, v_{i j}\right)_{j<i} \in \mathcal{L}_{\bullet}^{2}(\Sigma)$ is associated to the element $\mathcal{I}(\mathfrak{u})=\left(u_{0}, v_{0}, \cdots, u_{L}, v_{L}\right) \in \mathcal{S L}^{2}(\Sigma)$ according to

$$
\left(u_{i}, v_{i}\right)= \begin{cases}\left(u_{i j}, v_{i j}\right) & \text { on } \Gamma_{i j} \text { if } 0 \leq j<i \\ \left(u_{j i},-v_{j i}\right) & \text { on } \Gamma_{j i} \text { if } 0 \leq i<j, \quad i=0, \cdots, L . \\ \left(u_{i \bullet} \equiv 0, v_{i \bullet}\right) & \text { on } \Gamma_{i \bullet},\end{cases}
$$

In a similar manner, we find an isomorphism between $\mathcal{S L}^{2, \perp}(\Sigma)$ and $\mathcal{L}_{\bullet}^{2}(\Sigma)$. In comparison to (2.14), when going from $\mathcal{L}_{\bullet}^{2}(\Sigma)$ to $\mathcal{S} \mathcal{L}^{2, \perp}(\Sigma)$, the idea is to flip the role of Dirichlet data $u_{i j}$ and Neumann data $v_{i j}$. Any element $\mathfrak{u}=\left(u_{i j}, v_{i j}\right)_{j<i} \in \mathcal{L}_{\bullet}^{2}(\Sigma)$ is associated to the element $\mathcal{J}(\mathfrak{u})=\left(u_{0}, v_{0}, \cdots, u_{L}, v_{L}\right) \in \mathcal{S} \mathcal{L}^{2, \perp}(\Sigma)$ via

$$
\left(u_{i}, v_{i}\right)= \begin{cases}\left(-v_{i j}, u_{i j}\right) & \text { on } \Gamma_{i j} \text { if } 0 \leq j<i, \\ \left(v_{j i}, u_{j i}\right) & \text { on } \Gamma_{j i} \text { if } 0 \leq i<j, \quad i=0, \cdots, L . \\ \left(-v_{i \bullet}, u_{i \bullet} \equiv 0\right) & \text { on } \Gamma_{i \bullet},\end{cases}
$$

This leads to another Formulation equivalent to Formulation 2.2 with ansatz and test functions taken in $\mathcal{L}_{\bullet}^{2}(\Sigma)$, where $\mathcal{I}$ and $\mathcal{J}$ represent the action of the isomorphisms introduced above in (2.14) and (2.15), respectively.

Formulation 2.3 ([36, Form. 4.3.17]). Find $\mathfrak{u} \in \mathcal{L}_{\bullet}^{2}(\Sigma)$ such that

$$
\left\langle\left\langle\left(\mathrm{Id}-\mathbf{M}_{\Sigma}\right) \mathcal{I}(\mathfrak{u}), \mathcal{J}(\mathfrak{v})\right\rangle\right\rangle=\left\langle\left\langle\mathfrak{u}_{\text {inc }}, \mathcal{J}(\mathfrak{v})\right\rangle\right\rangle \quad \forall \mathfrak{v} \in \mathcal{L}_{\bullet}^{2}(\Sigma), \quad \mathfrak{u}_{\text {inc }}:=\gamma^{\Sigma} U_{\text {inc }}
$$


value distribution operator $\mathcal{I}$ at interface $\Gamma_{i j}$

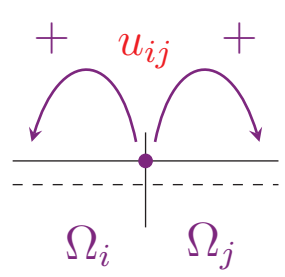

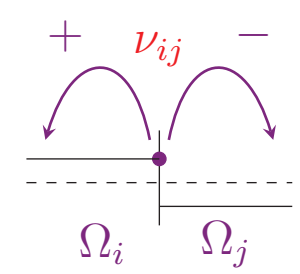

value distribution operator $\mathcal{J}$ at interface $\Gamma_{i j}$

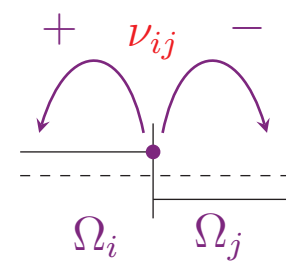

Figure 2: Visualization of the local action of the mappings $\mathcal{I}, \mathcal{J}$ at the interface $\Gamma_{i j}$ acting on an $L^{2}$ skeleton function $\mathfrak{u}_{i j}=\left(u_{i j}, v_{i j}\right) \in \mathcal{L}_{\bullet}^{2}(\Sigma)$. It is important to note the change of the role of Dirichlet and Neumann data inherent in the mapping $\mathcal{J}$.

\section{Combined field integral equations (CFIE)}

\subsection{Impenetrable scatterer $\left(L=0, \Omega_{\bullet} \neq \varnothing\right)$}

We first consider the case of a single impenetrable scatterer, i.e. $L=0$ and $\mathbb{R}^{d}=\Omega_{\bullet} \cup \partial \Omega_{\bullet} \cup$ $\Omega_{0}$. Then the second-kind BIE in Formulation 2.3 will fail to have a unique solution for infinitely many wave numbers $\kappa_{0}$ (spurious resonance phenomenon, [35, Section 3.9.2]). A widely used remedy are the so-called combined field integral equations based on an idea of Burton and Miller [3,4]. First, let us recall the direct CFIE policy for $L=0$. Later, in Subsection 3.2, we will adapt the idea to the case of a composite scatterer.

Let $U \in H_{0, \text { loc }}^{1}\left(\mathbb{R}^{d} \backslash \bar{\Omega}_{\bullet}\right)$ be a solution of an exterior Dirichlet problem, i.e. $U$ is assumed to solve (1.2) for $L=0$. We have seen that for $U$ equation (2.10) holds true. Taking the trace $\gamma^{0}$ of (2.10) yields:

$$
\left(\begin{array}{c}
\gamma_{D}^{0} U \\
\gamma_{N}^{0} U
\end{array}\right)=\left(\begin{array}{c}
\gamma_{D}^{0} G_{0}\left[\kappa_{0}\right]\left\{\gamma_{D}^{0} U, \gamma_{N}^{0} U\right\} \\
\gamma_{N}^{0} G_{\bullet}\left[\kappa_{0}\right]\left\{\gamma_{D}^{0} U, \gamma_{N}^{0} U\right\}
\end{array}\right)
$$

Using that $\gamma^{\bullet, c}=\left(\begin{array}{cc}1 & 0 \\ 0 & -1\end{array}\right) \gamma^{0}$ gives

$$
\begin{aligned}
\left(\begin{array}{l}
\gamma_{D}^{\bullet, c} U \\
\gamma_{N}^{\bullet, c} U
\end{array}\right) & =\left(\begin{array}{l}
\gamma_{D}^{\bullet, c} G_{0}\left[\kappa_{0}\right]\left\{\gamma_{D}^{0} U, \gamma_{N}^{0} U\right\} \\
\gamma_{N}^{\bullet, c} G_{0}\left[\kappa_{0}\right]\left\{\gamma_{D}^{0} U, \gamma_{N}^{0} U\right\}
\end{array}\right) \stackrel{(1.3)}{=}\left(\begin{array}{l}
\gamma_{D}^{\bullet, c} G_{0}\left[\kappa_{0}\right]\left\{\gamma_{D}^{\bullet, c} U,-\gamma_{N}^{\bullet, c} U\right\} \\
\gamma_{N}^{\bullet, c} G_{0}\left[\kappa_{0}\right]\left\{\gamma_{D}^{\bullet, c} U,-\gamma_{N}^{\bullet, c} U\right\}
\end{array}\right) \\
& =-\left(\begin{array}{l}
\gamma_{D}^{\bullet, c} G_{\bullet}\left[\kappa_{0}\right]\left\{\gamma_{D}^{\bullet, c} U, \gamma_{N}^{\bullet, c} U\right\} \\
\gamma_{N}^{\bullet, c} G_{\bullet}\left[\kappa_{0}\right]\left\{\gamma_{D}^{\bullet, c} U, \gamma_{N}^{\bullet, c} U\right\}
\end{array}\right),
\end{aligned}
$$

and thus

$$
-\gamma^{\bullet, c} U=\mathrm{P}_{\bullet, c}\left(\gamma^{\bullet, c} U\right)
$$

The operator $P_{\bullet, c}$ is known as Calderón projector (see [35, Proposition 3.6.2]). The first equation in (3.1) corresponds to the Dirichlet trace while the second equation is related 
to the Neumann trace. Sloppily speaking, taking just one of the two equations in (3.1) to characterize the boundary data at impenetrable objects means that we lose information.

We consider the second-kind BIE from Formulation 2.3 for $L=0$. Due to our choice of the test space $\mathcal{S} \mathcal{L}^{2, \perp}(\Sigma)=L^{2}\left(\partial \Omega_{0}\right) \times\{0\}$, we rely merely on the second equation in (3.1) which is related to the Neumann trace. However, in the case when $\kappa_{0}^{2}$ is a Neumann eigenvalue of $-\Delta$ in $\Omega_{\bullet}$, i.e. if

$$
\Delta U+\kappa_{0}^{2} U=0 \quad \text { in } \Omega_{\bullet}, \quad \gamma_{N}^{\bullet} U=0,
$$

has a nontrivial weak solution $U \in H_{0, \text { loc }}^{1}\left(\Omega_{\bullet}\right)$, this second equation will fail to have a unique solution. In this case, we have to make use of both equations in (3.2) to ensure that the resulting formulation is equivalent to (1.2), $L=0$.

The CFIE ansatz is to use a complex linear combination of the two equations in (3.1). For this purpose, we introduce the trace transformation operator $\Psi_{\eta}$ that transfers Dirichlet to Neumann data and multiplies them by i $\eta$, for some $\eta \in \mathbb{R} \backslash\{0\}$ :

$$
\Psi_{\eta}: L^{2}\left(\partial \Omega_{\bullet}\right) \times\{0\} \rightarrow\{0\} \times L^{2}\left(\partial \Omega_{\bullet}\right), \quad \mathfrak{v}=(v, 0) \mapsto \Psi_{\eta}(\mathfrak{v})=\mathrm{i} \eta(0, v) .
$$

Then, the direct combined field integral approach corresponding to Formulation 2.3 in the case $L=0$ boils down to the following variational problem:

Formulation 3.1 ([36, Form. 4.3.21]). Fix $\eta \in \mathbb{R} \backslash\{0\}$. Seek $\mathfrak{u} \in\{0\} \times L^{2}\left(\partial \Omega_{0}\right)$ such that for all $\mathfrak{v} \in L^{2}\left(\partial \Omega_{0}\right) \times\{0\}$, we have

$$
\left\langle\left\langle\left(\mathrm{Id}-\gamma^{0} \mathrm{G}_{0}\left[\kappa_{0}\right]\right) \mathfrak{u}, \Psi_{\eta}(\mathfrak{v})+\mathfrak{v}\right\rangle\right\rangle_{\mathcal{H}\left(\partial \Omega_{0}\right)}=\left\langle\left\langle\gamma^{0} U_{\text {inc }}, \Psi_{\eta}(\mathfrak{v})+\mathfrak{v}\right\rangle\right\rangle_{\mathcal{H}\left(\partial \Omega_{0}\right)}
$$

where the trace transformation operator $\Psi_{\eta}$ is defined in (3.4).

Splitting the boundary integral operator $\gamma^{0} \mathrm{G}_{0}\left[\kappa_{0}\right]$ into components acting on individual traces, we find that Formulation 3.1 agrees with the direct CFIE of $[3,4]$.

\subsection{Composite scatterer}

Now we return to the general situation of a scatterer with $L>0$ penetrable material domains and $\Omega_{\bullet} \neq \varnothing$. Our goal is to apply the same strategy as in Subsection 3.1 in the case of a composite scatterer. The idea is to add a generalized version of the first equation of (3.1), related to the Dirichlet trace, as an additional term to Formulation 2.3. This modification in the spirit of CFIEs can be expected to suppress spurious resonances caused by the presence of impenetrable objects.

Starting with the global representation formula (2.10) and using the identity (2.11), for a solution $U$ of (1.2) we obtain that

$$
\gamma_{D}^{\bullet, c}\left(U-\left.U_{\mathrm{inc}}\right|_{\Omega_{0}}\right)=\gamma_{D}^{\bullet, c} \mathbb{M}_{\Sigma}\left(\gamma^{\Sigma}\left(U-\left.U_{\mathrm{inc}}\right|_{\Omega_{0}}\right)\right)
$$




$$
\begin{array}{rrrl}
\Leftrightarrow & \gamma_{D}^{\bullet, c}\left(U-\left.U_{\text {inc }}\right|_{\Omega_{0}}-\mathbb{M}_{\Sigma}\left(\gamma^{\Sigma}\left(U-\left.U_{\text {inc }}\right|_{\Omega_{0}}\right)\right)\right) & =0 \\
\Leftrightarrow & \gamma_{D}^{\bullet, c}\left(-U+\mathbb{M}_{\Sigma}\left(\gamma^{\Sigma} U\right)+U_{\text {inc }}\right) & =0 .
\end{array}
$$

This equation represents the information we lost due to the choice of our test space $\mathcal{S L}^{2, \perp}(\Sigma)$ in Formulation 2.3, which has vanishing Neumann data on $\partial \Omega_{\text {• }}$. It corresponds to the generalized version of the first equation in (3.1). Our goal is to incorporate the information from (3.5) into Formulation 2.3 in order to suppress spurious resonances.

To introduce the extension to the setting of a composite scatterer of the trace transformation operator $\Psi_{\eta}$ from (3.4), we define the space $\widetilde{L^{2}}\left(\partial \Omega_{\bullet}\right)$, which can be seen as the extension of $L^{2}$-Dirichlet data associated with the impenetrable subdomain in $L^{2}\left(\partial \Omega_{\bullet}\right)$ to the skeleton space $\mathcal{L}^{2}(\Sigma)$ from Definition 2.4:

$$
\widetilde{L^{2}}\left(\partial \Omega_{\bullet}\right):=\left(\prod_{0 \leq j<i \leq L}\{0\} \times\{0\}\right) \times\left(\prod_{0 \leq i \leq L} L^{2}\left(\Gamma_{i}\right) \times\{0\}\right) .
$$

In order to make sense of the notation $\mathfrak{v}=\left(v_{i j}, \varphi_{i j}\right)_{j<i} \in \widetilde{L^{2}}\left(\partial \Omega_{\bullet}\right)$, we set $\bullet<j$ for all $j \in\{0, \cdots, L\}$. For any fixed $\eta \in \mathbb{R} \backslash\{0\}$ we define the trace transformation operator $\Psi_{\eta}: \mathcal{L}_{\bullet}^{2}(\Sigma) \rightarrow \widetilde{L^{2}}\left(\partial \Omega_{\bullet}\right) \subset \mathcal{L}^{2}(\Sigma)$,

$$
\mathfrak{v}=\left(v_{i j}, \varphi_{i j}\right)_{j<i} \mapsto \Psi_{\eta}(\mathfrak{v})=\left(\Psi_{\eta}(\mathfrak{v})\right)_{j<i}, \quad\left(\Psi_{\eta}(\mathfrak{v})\right)_{j<i}:= \begin{cases}\left(\mathrm{i} \eta \varphi_{i \bullet}, 0\right) & i=0, \cdots, L, \\ (0,0) & 0 \leq j<i \leq L .\end{cases}
$$

We may also need the straightforward extension of the isometry $\mathcal{J}$ defined in (2.15) to the space $\mathcal{L}^{2}(\Sigma)=\mathcal{L}_{\bullet}^{2}(\Sigma) \oplus \widetilde{L^{2}}\left(\partial \Omega_{\bullet}\right)$ from Definition 2.4. Namely, $\mathcal{J}(\mathfrak{u})=\left(u_{0}, v_{0}, \cdots, u_{L}, v_{L}\right) \in$ $\mathcal{M L}^{2}(\Sigma)$ is given by

$$
\left(u_{i}, v_{i}\right)= \begin{cases}\left(-v_{i j}, u_{i j}\right) & \text { on } \Gamma_{i j} \text { if } 0 \leq j<i \\ \left(v_{j i}, u_{j i}\right) & \text { on } \Gamma_{j i} \text { if } 0 \leq i<j, \quad i=0, \cdots, L \\ \left(-v_{i \bullet}, u_{i \bullet}\right) & \text { on } \Gamma_{i \bullet},\end{cases}
$$

Starting with the identity from (2.10) and (3.5) we obtain

$$
\begin{aligned}
&\left\langle\left\langle\left(\mathrm{Id}-\mathbf{M}_{\Sigma}\right)\left(\gamma^{\Sigma} U\right)-\gamma^{\Sigma} U_{\text {inc }}, \mathcal{J}\left(\Psi_{\eta}(\mathfrak{v})\right)\right\rangle\right\rangle \\
&=-\left\langle\left\langle-\gamma^{\Sigma} U+\mathbf{M}_{\Sigma}\left(\gamma^{\Sigma} U\right)+\gamma^{\Sigma} U_{\text {inc }}, \mathcal{J}\left(\Psi_{\eta}(\mathfrak{v})\right)\right\rangle\right\rangle \\
& \stackrel{(2.8)}{=}-\mathrm{i} \eta \sum_{0 \leq i \leq L} \int_{\Gamma_{i \bullet}} \gamma_{D}^{i}\left(-U+\mathbb{M}_{\Sigma}\left(\gamma^{\Sigma} U\right)+U_{\text {inc }}\right) \varphi_{i \bullet} \mathrm{d} S \\
&=-\mathrm{i} \eta \sum_{0 \leq i \leq L} \int_{\Gamma_{i \bullet}} \gamma_{D}^{\bullet, c}\left(-U+\mathbb{M}_{\Sigma}\left(\gamma^{\Sigma} U\right)+U_{\text {inc }}\right) \varphi_{i \bullet} \mathrm{d} S \stackrel{(3.5)}{=} 0,
\end{aligned}
$$


under application of the isometry $\mathcal{J}$ from (3.6) and using the fact that by definition of the traces in the beginning of Sect. 2.1 we have $\gamma_{D}^{i}=\gamma_{D}^{\bullet, c}$ on $\Gamma_{i \bullet}, i \in\{0,1, \cdots, L\}$.

Since equation (3.7) holds true for the solution $U$ of (1.2), the following formulation is still consistent with the transmission problem.

Formulation 3.2 ([36, Form. 4.3.22]). Find $\mathfrak{u} \in \mathcal{L}_{\bullet}^{2}(\Sigma)$ such that for all $\mathfrak{v} \in \mathcal{L}_{\bullet}^{2}(\Sigma)$

$$
\left\langle\left\langle\left(\mathrm{Id}-\mathbf{M}_{\Sigma}\right) \mathcal{I}(\mathfrak{u}), \mathcal{J}\left(\mathfrak{v}+\Psi_{\eta}(\mathfrak{v})\right)\right\rangle\right\rangle=\left\langle\left\langle\mathfrak{u}_{\text {inc }}, \mathcal{J}\left(\mathfrak{v}+\Psi_{\eta}(\mathfrak{v})\right)\right\rangle\right\rangle, \quad \mathfrak{u}_{\text {inc }}:=\gamma^{\Sigma} U_{\text {inc }} .
$$

Also in this case, uniqueness of solutions remains open, $c f$. Remark 2.1.

\section{Galerkin boundary element discretization of the second-kind formulations}

\subsection{Boundary element spaces}

For the Galerkin discretization of the variational boundary integral equations of Formulations 2.3 and 3.2 we have to rely on a finite-dimensional subspace $V_{M}$ of $\mathcal{L}_{\bullet}^{2}(\Sigma)$. We first give a general abstract description before discussing concrete choices.

Following the interface-oriented perspective of Definition 2.4 we decompose trial and test functions into interface contributions:

$$
\begin{aligned}
& \mathfrak{u}=\left(\left(u_{i j}, v_{i j}\right)_{0 \leq j<i \leq L},\left(0, v_{i \bullet}\right)_{0 \leq i \leq L}\right) \in \mathcal{L}_{\bullet}^{2}(\Sigma) \\
& \mathfrak{v}=\left(\left(v_{i j}, \varphi_{i j}\right)_{0 \leq j<i \leq L}\left(0, \varphi_{i \bullet}\right)_{0 \leq i \leq L}\right) \in \mathcal{L}_{\bullet}^{2}(\Sigma) .
\end{aligned}
$$

For the Galerkin discretization of (4.6), we rely on $M_{i j}$-dimensional, $M_{i j} \in \mathbb{N}$, subspaces of $L^{2}\left(\Gamma_{i j}\right)$ spanned by basis functions $b_{i j}^{1}, \cdots, b_{i j}^{M_{i j}}$, where $i \in\{0, \cdots, L\}, j \in\{\bullet, 0, \cdots, i-1\}$. This gives basis expansions of interface components $u_{i j}, v_{i j}, v_{i j}$, and $\varphi_{i j}$, of Galerkin trial and test functions $\mathfrak{u}, \mathfrak{v} \in V_{M}$, for instance,

$$
u_{i j}=\sum_{\ell=1}^{M_{i j}} u_{i j}^{\ell} b_{i j}^{\ell} \quad \text { with } \quad \overrightarrow{\boldsymbol{u}}_{i j}:=\left(u_{i j}^{1}, \cdots, u_{i j}^{M_{i j}}\right)^{\top} \in \mathbb{C}^{M_{i j}} .
$$

Wherever required, the basis functions will be considered as elements of $L^{2}(\Sigma)$ after extension by zero. The resulting subspace $V_{M} \subset \mathcal{L}_{\bullet}^{2}(\Sigma)$ has dimension

$$
M:=2 \sum_{0 \leq j<i \leq L} M_{i j}+\sum_{0 \leq i \leq L} M_{i \bullet}
$$


We denote the vectors of the basis expansion coefficients of $\mathfrak{u}, \mathfrak{v} \in V_{M}$ as

$$
\begin{aligned}
& \overrightarrow{\mathfrak{u}}=\left(\left(\overrightarrow{\boldsymbol{u}}_{i j}, \overrightarrow{\boldsymbol{v}}_{i j}\right)_{0 \leq j<i \leq L}\left(\overrightarrow{\boldsymbol{v}}_{i \bullet}\right)_{0 \leq i \leq L}\right) \in \mathbb{C}^{M}, \\
& \overrightarrow{\mathfrak{v}}=\left(\left(\overrightarrow{\boldsymbol{v}}_{i j}, \overrightarrow{\boldsymbol{\varphi}}_{i j}\right)_{0 \leq j<i \leq L,}\left(\overrightarrow{\boldsymbol{\varphi}}_{i \bullet}\right)_{0 \leq i \leq L}\right) \in \mathbb{C}^{M} .
\end{aligned}
$$

Of course, we have dropped the vanishing Dirichlet contribution on $\Gamma_{i \bullet}$. The local coefficient vectors $\overrightarrow{\boldsymbol{u}}_{i j}, \overrightarrow{\boldsymbol{v}}_{i j}, \overrightarrow{\boldsymbol{v}}_{i j}$ and $\overrightarrow{\boldsymbol{\varphi}}_{i j}$ are defined as in (4.2).

In the concrete case of fixed-degree piecewise polynomial boundary element spaces $V_{M}$ we rely on a mesh/triangulation $\mathcal{T}=\left\{\tau_{1}, \cdots, \tau_{|\mathcal{T}|}\right\}$ of $\Sigma$ (see [35, Sect. 4.1.2]) that resolves the given geometry of $\Sigma$, in the sense that the closure of every $\Gamma_{i j}$ agrees with the union of some closed cells of $\mathcal{T}$. Mesh based boundary element subspaces $V_{M} \subset \mathcal{L}_{\bullet}^{2}(\Sigma)$ do not have to satisfy any continuity conditions at cell boundaries. Therefore, we opt for a simple piecewise polynomial discontinuous approximation of $\mathcal{L}_{\bullet}^{2}(\Sigma)$ by means of $\|$

$$
V_{M}:=V_{\mathcal{T}, p}:=\mathcal{S}_{\mathcal{T}}^{p,-1}(\Sigma) \times \mathcal{S}_{\mathcal{T}}^{p,-1}(\Sigma) \subset \mathcal{L}_{\bullet}^{2}(\Sigma),
$$

where the total degree $p \in \mathbb{N}_{0}$ is fixed, see [35, Def. 4.1.17] $(d=3)$, [37, Sect. 10.2] $(d=2)$. In other words, we use piecewise polynomial functions of maximal total degree $p$ in each mesh cell. $\mathcal{L}_{\bullet}^{2}(\Sigma)$-stable bases of $V_{\mathcal{T}, p}$ are readily available. In the case $p=0$ we simply use the set of characteristic functions of the mesh elements $\tau_{k} \in \mathcal{T}$.

\subsection{Galerkin linear systems of equations}

In this section, we dip into the details of the Galerkin boundary element discretization of Formulation 2.3. For the sake of lucidity, we restrict ourselves to the situation depicted in Fig. 3. This situation is sufficiently general to convey all key considerations.

The main idea is to consider all the operator contributions from Formulation 2.3 interface-wise instead of subdomain-wise, as it has already been done in the definition of the $L^{2}$ single-trace space $\mathcal{S} \mathcal{L}^{2}(\Sigma)$ (see Definition 2.2) and the $L^{2}$ skeleton trace space $\mathcal{L}_{\bullet}^{2}(\Sigma)$ (see Definition 2.4). Definition 2.2 of the single-trace space and the isometry given in (2.14) adopt the convention that at transmission interfaces the intrinsic orientation chosen for the interface $\Gamma_{i j}$ is inherited by the orientation of the boundary of the adjacent domain $\Omega_{i}$ with larger index $i>j$. In the case of an impenetrable interface $\Gamma_{i \bullet} \subset \Omega_{\bullet}$ the orientation is induced by the penetrable domain $\Omega_{i}, i \in\{0,1, \cdots, L\}$. In Fig. 3, the intrinsic orientations of the interfaces $\Gamma_{01}, \Gamma_{0}$ and $\Gamma_{1}$. are indicated through the directions of the normal vectors $\mathbf{n}_{01}, \mathbf{n}_{0 \bullet}$ and $\mathbf{n}_{1 \bullet}$, respectively.

Based on this convention, we study the structure of Formulation 2.3:

Seek $\mathfrak{u} \in \mathcal{L}_{\bullet}^{2}(\Sigma)$ such that

$$
\left\langle\left\langle\left(\mathrm{Id}-\mathbf{M}_{\Sigma}\right) \mathcal{I}(\mathfrak{u}), \mathcal{J}(\mathfrak{v})\right\rangle\right\rangle=\left\langle\left\langle\mathfrak{u}_{\text {inc }}, \mathcal{J}(\mathfrak{v})\right\rangle\right\rangle \forall \mathfrak{v} \in \mathcal{L}_{\bullet}^{2}(\Sigma) .
$$

$\|$ The notations for boundary element spaces are borrowed from [35, Sect. 4.1]. 


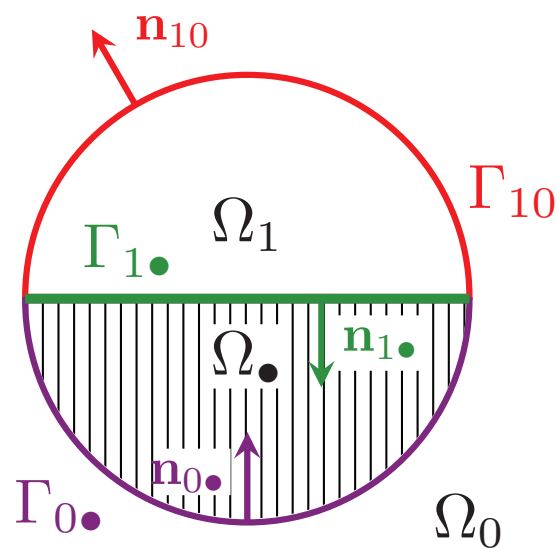

Figure 3: Model geometry $(L=1)$ for studying the implementation of the second-kind formulation.

To begin with, we study the discretization of the identity operator. We start from (2.8)

$$
\langle\langle\mathfrak{u}, \mathfrak{v}\rangle\rangle=\sum_{0 \leq j<i \leq L} \int_{\Gamma_{i j}} u_{i} \varphi_{i}-v_{i} v_{i}+u_{j} \varphi_{j}-v_{j} v_{j} \mathrm{~d} S+\sum_{0 \leq i \leq L} \int_{\Gamma_{i \bullet}} u_{i} \varphi_{i}-v_{i} v_{i} \mathrm{~d} S,
$$

and replace $\mathfrak{u} \leftarrow \mathcal{I}(\mathfrak{u}), \mathfrak{v} \leftarrow \mathcal{J}(\mathfrak{v})$. In light of the definitions (2.14) and (2.15) of $\mathcal{I}$ and $\mathcal{J}$, respectively, this boils down to setting in the first sum of (2.8) $u_{i} \leftarrow u_{i j}, \varphi_{i} \leftarrow v_{i j}, v_{i} \leftarrow v_{i j}$, $v_{i} \leftarrow-\varphi_{i j}, u_{j} \leftarrow u_{i j}, \varphi_{j} \leftarrow v_{i j}, v_{j} \leftarrow-v_{i j}$, and $v_{j} \leftarrow \varphi_{i j}$. In the second sum we replace $u_{i} \leftarrow 0$, $\varphi_{i} \leftarrow 0, v_{i} \leftarrow v_{i \bullet}$, and $v_{i} \leftarrow-\varphi_{i \bullet}$. This yields

$$
\langle\langle\mathcal{I}(\mathfrak{u}), \mathcal{J}(\mathfrak{v})\rangle\rangle=2 \sum_{0 \leq j<i \leq L} \int_{\Gamma_{i j}} u_{i j} v_{i j}+v_{i j} \varphi_{i j} \mathrm{~d} S+\sum_{0 \leq i \leq L} \int_{\Gamma_{i \bullet}} v_{i \bullet} \varphi_{i \bullet} \mathrm{d} S
$$

Thus, after introducing the interface mass matrices

$$
\mathbf{M}_{i j}^{i j}:=\left(\int_{\Gamma_{i j}} b_{i j}^{\ell} b_{i j}^{k} \mathrm{~d} S\right)_{1 \leq \ell, k \leq M_{i j}} \in \mathbb{R}^{M_{i j}, M_{i j}},
$$

in the model situation of Fig. 3 the bilinear form $(\mathfrak{u}, \mathfrak{v}) \mapsto\langle\langle\mathcal{I}(\mathfrak{u}), \mathcal{J}(\mathfrak{v})\rangle\rangle$ gives rise to the block-diagonal Galerkin matrix

$$
\left(\begin{array}{cccc}
2 \mathbf{M}_{10}^{10} & \mathbf{0} & \mathbf{0} & \mathbf{0} \\
\mathbf{0} & 2 \mathbf{M}_{10}^{10} & \mathbf{0} & \mathbf{0} \\
\mathbf{0} & \mathbf{0} & \mathbf{M}_{0 \bullet \bullet}^{0 \bullet} & \mathbf{0} \\
\mathbf{0} & \mathbf{0} & \mathbf{0} & \mathbf{M}_{1 \bullet \bullet}^{1 \bullet}
\end{array}\right),
$$

where the color code of Fig. 3 is used to highlight contributions of particular interfaces. The color red denotes the interface $\Gamma_{10}$, violet stands for $\Gamma_{0}$ and green represents $\Gamma_{1} \bullet$ 
Next, we examine the remaining term on the left hand side in (4.6). We continue using the interface-wise notation (4.1) for components of $\mathfrak{u}, \mathfrak{v} \in \mathcal{L}_{\bullet}^{2}(\Sigma)$. As above we write the expressions interface-wise using the definition of the duality pairing from (2.8). We also rely on the insight that, if we restrict $\mathbf{M}_{\Sigma}$ to $\mathcal{S} \mathcal{L}^{2}(\Sigma)$, then Range $\left(\mathbf{M}_{\Sigma}\right)$ will be singlevalued.

$$
\begin{array}{r}
\left\langle\left\langle\mathbf{M}_{\Sigma} \mathcal{I}(\mathfrak{u}), \mathcal{J}(\mathfrak{v})\right\rangle\right\rangle=2 \sum_{0 \leq j<i \leq L} \int_{\Gamma_{i j}} \gamma_{D}^{i} \mathbb{M}_{\Sigma}(\mathcal{I}(\mathfrak{u})) v_{i j}-\gamma_{N}^{i} \mathbb{M}_{\Sigma}(\mathcal{I}(\mathfrak{u}))\left(-\varphi_{i j}\right) \mathrm{d} S \\
+\sum_{0 \leq i \leq L} \int_{\Gamma_{i} \bullet} 0-\gamma_{N}^{i} \mathbb{M}_{\Sigma}(\mathcal{I}(\mathfrak{u}))\left(-\varphi_{i j}\right) \mathrm{d} S
\end{array}
$$

If we translate this to the concrete setting of Fig. 3, we get

$$
\begin{aligned}
& \left\langle\left\langle\mathbf{M}_{\Sigma} \mathcal{I}(\mathfrak{u}), \mathcal{J}(\mathfrak{v})\right\rangle\right\rangle=2 \int_{\Gamma_{10}} \gamma_{D}^{1} \mathbb{M}_{\Sigma}(\mathcal{I}(\mathfrak{u})) v_{10}+\gamma_{N}^{1} \mathbb{M}_{\Sigma}(\mathcal{I}(\mathfrak{u})) \varphi_{10} \mathrm{~d} S+
\end{aligned}
$$

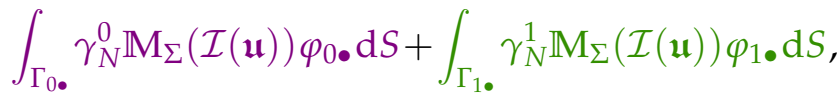

where the colors indicate to which interface a term contributes (see Fig. 3).

Next, we split the trial space into interface contributions and take into account that at the transmission interface $\Gamma_{10}$, we have two adjacent domains contributing to the interface, while for the Dirichlet interfaces $\Gamma_{0}$ and $\Gamma_{1} \bullet$, we have only one contribution from the penetrable side $\Omega_{0}$ and $\Omega_{1}$, respectively. Throughout, we interpret the interface-wise defined functions $u_{i j}, v_{i j}$ and $v_{i j}, \varphi_{i j}, i, j \in\{\bullet, 0,1, \cdots, L\}$ as functions on the whole skeleton $\Sigma$ after formal extension by 0 . Nine terms result from splitting trial and test functions into three interface contributions each.

$$
\begin{aligned}
& \left\langle\left\langle\mathbf{M}_{\Sigma} \mathcal{I}(\mathfrak{u}), \mathcal{J}(\mathfrak{v})\right\rangle\right\rangle=2 \int_{\Gamma_{10}} \gamma_{D}^{1}\left(\mathbb{G}_{1}\left[\kappa_{1}\right]\right)\left(u_{10}, v_{10}\right) v_{10}+\gamma_{D}^{1}\left(\mathbb{G}_{0}\left[\kappa_{0}\right]\right)\left(u_{10},-v_{10}\right) v_{10} \\
& +\gamma_{N}^{1}\left(\mathbb{G}_{1}\left[\kappa_{1}\right]\right)\left(u_{10}, v_{10}\right) \varphi_{10}+\gamma_{N}^{1}\left(\mathbb{G}_{0}\left[\kappa_{0}\right]\right)\left(u_{10},-v_{10}\right) \varphi_{10} \mathrm{~d} S \\
& +2 \int_{\Gamma_{10}} \gamma_{D}^{1}\left(\mathbb{G}_{0}\left[\kappa_{0}\right]\right)\left(0, v_{0} \bullet v_{10} \mathrm{~d} S+2 \int_{\Gamma_{10}} \gamma_{D}^{1}\left(\mathbb{G}_{1}\left[\kappa_{1}\right]\right)\left(0, v_{1} \bullet v_{10} \mathrm{~d} S\right.\right. \\
& +2 \int_{\Gamma_{10}} \gamma_{N}^{1}\left(\mathbb{G}_{0}\left[\kappa_{0}\right]\right)\left(0, v_{0} \bullet \varphi_{10} \mathrm{~d} S+2 \int_{\Gamma_{10}} \gamma_{N}^{1}\left(\mathbb{G}_{1}\left[\kappa_{1}\right]\right)\left(0, v_{1} \bullet \varphi_{10} \mathrm{~d} S\right.\right. \\
& +\int_{\Gamma_{0} \bullet} \gamma_{N}^{0}\left(\mathbb{G}_{1}\left[\kappa_{1}\right]\right)\left(u_{10}, v_{10}\right) \varphi_{0 \bullet}+\gamma_{N}^{0}\left(\mathbb{G}_{0}\left[\kappa_{0}\right]\right)\left(u_{10},-v_{10}\right) \varphi_{0} \mathrm{~d} S
\end{aligned}
$$

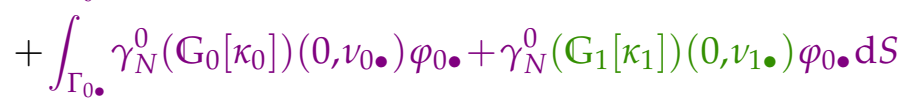

$$
\begin{aligned}
& +\int_{\Gamma_{1} \bullet} \gamma_{N}^{1}\left(\mathbb{G}_{1}\left[\kappa_{1}\right]\right)\left(u_{10}, v_{10}\right) \varphi_{1} \bullet+\gamma_{N}^{1}\left(\mathbb{G}_{0}\left[\kappa_{0}\right]\right)\left(u_{10},-v_{10}\right) \varphi_{1} \bullet d S
\end{aligned}
$$

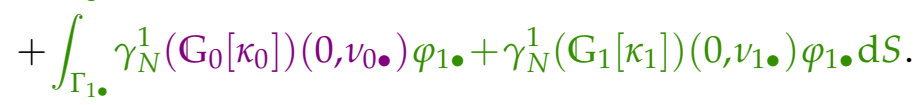


In a next step, at the transmission interface $\Gamma_{10}$, we rewrite the potential that is related to $\Omega_{0}$. By definition of the normal $\mathbf{n}_{1}=-\mathbf{n}_{0}$, we have that

$$
\mathbb{G}_{0}\left[\kappa_{0}\right]\left(u_{10}, v_{10}\right)=-\mathbb{G}_{1}\left[\kappa_{0}\right]\left(u_{10},-v_{10}\right),
$$

and, therefore, find

$$
\begin{aligned}
& \left\langle\left\langle\mathbf{M}_{\Sigma} \mathcal{I}(\mathfrak{u}), \mathcal{J}(\mathfrak{v})\right\rangle\right\rangle=2 \int_{\Gamma_{10}} \gamma_{D}^{1}\left(\mathbb{G}_{1}\left[\kappa_{1}\right]-\mathbb{G}_{1}\left[\kappa_{0}\right]\right)\left(u_{10}, v_{10}\right) v_{10} \\
& +\gamma_{N}^{1}\left(\mathbb{G}_{1}\left[\kappa_{1}\right]-\mathbb{G}_{1}\left[\kappa_{0}\right]\right)\left(u_{10}, v_{10}\right) \varphi_{10} \mathrm{~d} S \\
& +2 \int_{\Gamma_{10}} \gamma_{D}^{1}\left(\mathbb{G}_{0}\left[\kappa_{0}\right]\right)\left(0, v_{0} \bullet\right) v_{10} \mathrm{~d} S+2 \int_{\Gamma_{10}} \gamma_{D}^{1}\left(\mathbb{G}_{1}\left[\kappa_{1}\right]\right)\left(0, v_{1} \bullet v_{10} \mathrm{~d} S\right. \\
& +2 \int_{\Gamma_{10}} \gamma_{N}^{1}\left(\mathbb{G}_{0}\left[\kappa_{0}\right]\right)\left(0, v_{0} \bullet \varphi_{10} \mathrm{~d} S+2 \int_{\Gamma_{10}} \gamma_{N}^{1}\left(\mathbb{G}_{1}\left[\kappa_{1}\right]\right)\left(0, v_{1} \bullet \varphi_{10} \mathrm{~d} S\right.\right. \\
& +\int_{\Gamma_{0} \bullet} \gamma_{N}^{0}\left(\mathbb{G}_{1}\left[\kappa_{1}\right]-\mathbb{G}_{1}\left[\kappa_{0}\right]\right)\left(u_{10}, v_{10}\right) \varphi_{0} \cdot \mathrm{d} S
\end{aligned}
$$

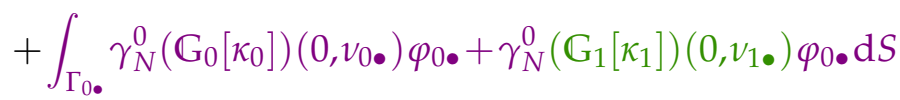

$$
\begin{aligned}
& +\int_{\Gamma_{1} \bullet} \gamma_{N}^{1}\left(\mathbb{G}_{1}\left[\kappa_{1}\right]-\mathbb{G}_{1}\left[\kappa_{0}\right]\right)\left(u_{10}, v_{10}\right) \varphi_{1} \bullet \mathrm{d} S
\end{aligned}
$$

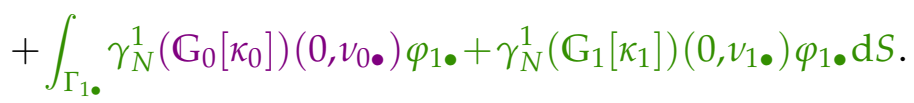

Finally, we have derived an interface-wise representation of Formulation 2.3.

Now we proceed with the Galerkin discretization based on basis expansions of all trial and test functions as introduced above, see (4.2). Our arrangement of basis functions leads to the following block partitioned Galerkin matrix:

$$
\left(\begin{array}{lll}
\mathbf{C}_{10}^{10} & \mathbf{C}_{0}^{10} & \mathbf{C}_{1 \bullet}^{10} \\
\mathbf{C}_{10}^{0 \bullet} & \mathbf{C}_{0}^{0}: & \mathbf{C}_{1}^{0 \bullet} \\
\mathbf{C}_{10}^{10} & \mathbf{C}_{0 \bullet}^{1}: & \mathbf{C}_{1 \bullet}^{1}:
\end{array}\right) \in \mathbb{C}^{M, M}, \quad M:=2 M_{10}+M_{0 \bullet}+M_{1 \bullet} .
$$

The detailed structure of the matrix blocks is

$$
\mathbf{C}_{10}^{10}=2\left(\begin{array}{cc}
-\left(\mathbf{K}_{10}^{10}\left[\kappa_{1}\right]-\mathbf{K}_{10}^{10}\left[\kappa_{0}\right]\right) & \mathbf{V}_{10}^{10}\left[\kappa_{1}\right]-\mathbf{V}_{10}^{10}\left[\kappa_{0}\right] \\
\mathbf{W}_{10}^{10}\left[\kappa_{1}\right]-\mathbf{W}_{10}^{10}\left[\kappa_{0}\right] & \mathbf{K}_{10}^{\prime 10}\left[\kappa_{1}\right]-\mathbf{K}_{10}^{\prime 10}\left[\kappa_{0}\right]
\end{array}\right),
$$

which amounts to the difference of two Calderón operators(see [35, equation (3.122)]), where

$$
\mathbf{K}_{10}^{10}\left[\kappa_{1}\right]-\mathbf{K}_{10}^{10}\left[\kappa_{0}\right]:=\left(\int_{\Gamma_{10}} \gamma_{D}^{1}\left(\mathbb{D}_{1}\left[\kappa_{1}\right]-\mathbb{D}_{1}\left[\kappa_{0}\right]\right)\left(b_{10}^{j}\right) b_{10}^{i} \mathrm{~d} S\right)_{i, j=1}^{M_{10}} \in \mathbb{C}^{M_{10}, M_{10}}
$$

represents the difference of two double layer operators (see [35, Sect. 3.3.3]). The difference of two single layer operators (c.f. $[35,3.3 .2]$ ) gives rise to the matrix

$$
\mathbf{V}_{10}^{10}\left[\kappa_{1}\right]-\mathbf{V}_{10}^{10}\left[\kappa_{0}\right]:=\left(\int_{\Gamma_{10}} \gamma_{D}^{1}\left(\mathbb{S}_{1}\left[\kappa_{1}\right]-\mathbb{S}_{1}\left[\kappa_{0}\right]\right)\left(b_{10}^{j}\right) b_{10}^{i} \mathrm{~d} S\right)_{i, j=1}^{M_{10}} \in \mathbb{C}^{M_{10}, M_{10}}
$$


and the difference of two hypersingular operators (c.f. [35, Sect. 3.3.4]) and two adjoint double layer operators (see [35, Sect. 3.3.3]), respectively, leads to the matrices

$$
\mathbf{W}_{10}^{10}\left[\kappa_{1}\right]-\mathbf{W}_{10}^{10}\left[\kappa_{0}\right]:=\left(-\int_{\Gamma_{10}} \gamma_{N}^{1}\left(\mathbb{D}_{1}\left[\kappa_{1}\right]-\mathbb{D}_{1}\left[\kappa_{0}\right]\right)\left(b_{10}^{j}\right) b_{10}^{i} \mathrm{~d} S\right)_{i, j=1}^{M_{10}},
$$

and

$$
\mathbf{K}_{10}^{\prime 10}\left[\kappa_{1}\right]-\mathbf{K}_{10}^{\prime 10}\left[\kappa_{0}\right]:=\left(\int_{\Gamma_{10}} \gamma_{N}^{1}\left(\mathbb{S}_{1}\left[\kappa_{1}\right]-\mathbb{S}_{1}\left[\kappa_{0}\right]\right)\left(b_{10}^{j}\right) b_{10}^{i} \mathrm{~d} S\right)_{i, j=1}^{M_{10}} \in \mathbb{C}^{M_{10}, M_{10}}
$$

Obviously, at transmission interfaces we end up with differences of the classical kernels**. Taking the difference of two kernels leads to cancellation of leading singularities such that the integrals involved in $\mathbf{C}_{10}^{10}$ are at most weakly singular. They are amenable to the usual singular integration techniques from [35, Chapter 5], though we point out that numerical cancellation has to be avoided as discussed in [12, Page 51/52]. In short, one should implement a specific kernel for subtracted operators and use cancellationfree expressions. In our code, we use Taylor expansions to achieve a numerically stable implementation (see [12, eq. (5.7)-(5.9)]).

The next block is a coupling term of the Dirichlet interface $\Gamma_{0}$ and the transmission interface $\Gamma_{10}$ :

$$
\mathbf{C}_{0 \bullet}^{10}=2\left(\begin{array}{l}
\left(\int_{\Gamma_{10}} \gamma_{D}^{1}\left(\mathrm{~S}_{0}\left[\kappa_{0}\right]\right)\left(b_{0 \bullet}^{j}\right) b_{10}^{i} \mathrm{~d} S\right)_{\substack{1 \leq i \leq M_{10} \\
1 \leq j \leq M_{0}}} \\
\left(\int_{\Gamma_{10}} \gamma_{N}^{1}\left(\mathrm{~S}_{0}\left[\kappa_{0}\right]\right)\left(b_{0 \bullet}^{j}\right) b_{10}^{i} \mathrm{~d} S\right)_{\substack{1 \leq i \leq M_{10} \\
1 \leq j \leq M_{0}}}
\end{array}\right)=\left(\begin{array}{c}
\mathbf{V}_{0 \cdot \bullet}^{10}\left[\kappa_{0}\right] \\
\mathbf{K}_{0 \bullet}^{\prime 10}\left[\kappa_{0}\right]
\end{array}\right) .
$$

In this case, no cancellation occurs since at the Dirichlet boundary we only have an operator contribution from the penetrable subdomain $\Omega_{0}$. The kernel of the boundary integral operator $\gamma_{D}^{1}\left(\mathrm{~S}_{0}\left[\kappa_{0}\right]\right)$ is weakly singular, while the kernel of the operator $\gamma_{N}^{1}\left(\mathrm{~S}_{0}\left[\kappa_{0}\right]\right)$, i.e. the adjoint double layer operator $\mathbf{K}_{0 \bullet}^{\prime 10}$, has a strong singularity behaving like $\mathcal{O}\left(\frac{1}{\|x-y\|}\right)$ for $d=2$ and $\mathcal{O}\left(\frac{1}{\|x-y\|^{2}}\right)$ for $d=3$, respectively. Therefore, we face a principal part integral, which finally leads to a jump term $\frac{1}{2}$ Id on $\Gamma_{0 \bullet}$, when crossing the interface $\Gamma_{0 \bullet}$ (see [28, Sec. 7.2]). In the case of $\mathbf{C}_{0 \bullet}^{10}$, we can neglect this term because our trial functions are supported on the interface $\Gamma_{10}$, which only shares a set of measure zero with $\Gamma_{0 \bullet}$.

The same applies to the third block in the first row of (4.7),

$$
\mathbf{C}_{1 \bullet}^{10}=2\left(\begin{array}{l}
\left(\int_{\Gamma_{10}} \gamma_{D}^{1}\left(\mathbb{S}_{1}\left[\kappa_{1}\right]\right)\left(b_{1 \bullet}^{j}\right) b_{10}^{i} \mathrm{~d} S\right)_{\substack{1 \leq i \leq M_{10} \\
1 \leq j \leq M_{1}}} \\
\left(\int_{\Gamma_{10}} \gamma_{N}^{1}\left(\mathbb{S}_{1}\left[\kappa_{1}\right]\right)\left(b_{1 \bullet}^{j}\right) b_{10}^{i} \mathrm{~d} S\right)_{\substack{1 \leq i \leq M_{10} \\
1 \leq j \leq M_{1} \bullet}}
\end{array}\right)=\left(\begin{array}{c}
\mathbf{V}_{0 \bullet}^{10}\left[\kappa_{0}\right] \\
\mathbf{K}_{0 \bullet}^{\prime 10}\left[\kappa_{0}\right]
\end{array}\right) .
$$

\footnotetext{
** The operators associated with transmission interfaces are studied in great detail in [12, Lem. 5.3].
} 
The blocks in the second row of (4.7) are spawned by test functions supported on $\Gamma_{0 \text { e. }}$. Since the first block $\mathbf{C}_{10}^{0 \bullet}$ is associated with trial functions supported on the transmission interface $\Gamma_{10}$, we again obtain more regular differences of classical kernels, which are, at worst, weakly singular.

$$
\begin{aligned}
\mathbf{C}_{10}^{0 \bullet} & =\left(\begin{array}{c}
\left(-\int_{\Gamma_{0} \bullet} \gamma_{N}^{0}\left(\mathbb{D}_{1}\left[\kappa_{1}\right]-\mathbb{D}_{1}\left[\kappa_{0}\right]\right)\left(b_{10}^{j}\right) b_{0 \bullet}^{i} \mathrm{~d} S\right)_{\substack{1 \leq i \leq M_{0} \bullet \\
1 \leq j \leq M_{10}}}\left(\int_{\Gamma_{0} \bullet} \gamma_{N}^{0}\left(\mathrm{~S}_{1}\left[\kappa_{1}\right]-\mathrm{S}_{1}\left[\kappa_{0}\right]\right)\left(b_{10}^{j}\right) b_{0 \bullet}^{i} \mathrm{~d} S\right)_{\substack{1 \leq i \leq M_{0} \bullet \\
1 \leq j \leq M_{10}}}^{\top}
\end{array}\right)^{\top}=\left(\mathbf{W}_{10}^{0 \bullet}\left[\kappa_{1}\right]-\mathbf{W}_{10}^{0 \bullet}\left[\kappa_{0}\right] \quad \mathbf{K}_{10}^{\prime \bullet}\left[\kappa_{1}\right]-\mathbf{K}_{10}^{\prime \bullet \bullet}\left[\kappa_{0}\right]\right) .
\end{aligned}
$$

The second block $\mathrm{C}_{0 \bullet}^{0 \bullet}$ in the second row of (4.7) is the self-interaction of $\Gamma_{0 \bullet}$. As mentioned above, since the kernel $\gamma_{N, x}^{0} \gamma_{D, y}^{0} \Phi_{\kappa_{0}}(x, y)$ of $\gamma_{N}^{0}\left(\mathbb{S}_{0}\left[\mathcal{K}_{0}\right]\right)$ has a strong singularity in $\boldsymbol{x}=\boldsymbol{y}$, we obtain a contribution $\frac{1}{2}$ Id due to the principal part integral when crossing the interface $\Gamma_{0}$. After Galerkin discretization, this jump term is represented by the interface mass matrix $\mathbf{M}_{0 \bullet}^{0 \bullet}:=\left(\int_{\Gamma_{0} \bullet} b_{0 \bullet}^{j} b_{0 \bullet}^{i} \mathrm{~d} S\right)_{i, j=1}^{M_{0} \bullet}$. Thus, we obtain

$$
\mathbf{C}_{0 \bullet}^{0 \bullet}=\left(\int_{\Gamma_{0} \bullet} \gamma_{N}^{0}\left(\mathrm{~S}_{0}\left[\kappa_{0}\right]\right)\left(b_{0 \bullet}^{j}\right) b_{0 \bullet}^{i} \mathrm{~d} S\right)_{i, j=1}^{M_{0} \bullet}=\frac{1}{2} \mathbf{M}_{0 \bullet}^{0 \bullet}+\mathbf{K}_{0 \bullet}^{\prime 0 \bullet}\left[\kappa_{0}\right],
$$

as in the case of the second-kind formulation of the exterior Dirichlet problem. The last block $\mathbf{C}_{1}^{0 \bullet}$ in the second row has the form

$$
\mathbf{C}_{1 \bullet}^{0 \bullet}=\left(\int_{\Gamma_{0} \bullet} \gamma_{N}^{0}\left(S_{1}\left[\kappa_{1}\right]\right)\left(b_{1 \bullet}^{j}\right) b_{0 \bullet}^{i} \mathrm{~d} S\right)_{\substack{1 \leq i \leq M_{0} \bullet \\ 1 \leq j \leq M_{1} \bullet}}=\mathbf{K}_{1 \bullet}^{\prime 0} \bullet\left[\kappa_{1}\right] .
$$

Finally, the last row of the block matrix in (4.7) can be obtained analogously to the second row blocks.

$$
\begin{aligned}
& \mathbf{C}_{10}^{1 \bullet}=\left(\begin{array}{c}
\left(-\int_{\Gamma_{1} \bullet} \gamma_{N}^{1}\left(\mathbb{D}_{1}\left[\kappa_{1}\right]-\mathbb{D}_{1}\left[\kappa_{0}\right]\right)\left(b_{10}^{j}\right) b_{1 \bullet}^{i} \mathrm{~d} S\right)_{\substack{1 \leq i \leq M_{1} \bullet \\
1 \leq j \leq M_{10}}}\left(\int_{\Gamma_{0} \bullet} \gamma_{N}^{1}\left(\mathrm{~S}_{1}\left[\kappa_{1}\right]-\mathrm{S}_{1}\left[\kappa_{0}\right]\right)\left(b_{10}^{j}\right) b_{1 \bullet}^{i} \mathrm{~d} S\right)_{\substack{1 \leq i \leq M_{1} \bullet \\
1 \leq j \leq M_{10}}}
\end{array}\right)^{\top} \\
&=\left(\mathbf{W}_{10}^{1 \bullet}\left[\kappa_{1}\right]-\mathbf{W}_{10}^{1 \bullet}\left[\kappa_{0}\right] \quad \mathbf{K}_{10}^{\prime \bullet \bullet}\left[\kappa_{1}\right]-\mathbf{K}_{10}^{\prime 1 \bullet}\left[\kappa_{0}\right]\right), \\
& \mathbf{C}_{0 \bullet}^{1 \bullet}=\left(\int_{\Gamma_{1} \bullet} \gamma_{N}^{1}\left(\mathrm{~S}_{0}\left[\kappa_{0}\right]\right)\left(b_{0 \bullet}^{j}\right) b_{1 \bullet}^{i} \mathrm{~d} S\right)_{\substack{1 \leq i \leq M_{1} \bullet \\
1 \leq j \leq M_{0} \bullet}}=\mathbf{K}_{0 \bullet}^{\prime 1 \bullet}\left[\kappa_{0}\right], \\
& \mathbf{C}_{1 \bullet}^{1 \bullet}:=\left(\int_{\Gamma_{0} \bullet} \gamma_{N}^{1}\left(S_{1}\left[\kappa_{1}\right]\right)\left(b_{1 \bullet}^{j}\right) b_{0 \bullet}^{i} \mathrm{~d} S\right)_{i, j=1}^{M_{0} \bullet}=\frac{1}{2} \mathbf{M}_{1 \bullet}^{1 \bullet}+\mathbf{K}_{1 \bullet}^{\prime 1 \bullet}\left[\kappa_{1}\right] .
\end{aligned}
$$

Using the interface-wise decomposition in (4.7), we finally observe the following block structure for the linear system of equations arising from the Galerkin discretization of Formulation 2.3 in the case of the geometry depicted in Fig. 3 and choosing basis 
functions as described above in Section 4.1 [36, Form. 4.4.5]:

$$
\begin{aligned}
& \left(\begin{array}{cccc}
2 \mathbf{M}_{10}^{10} & \mathbf{0} & \mathbf{0} & \mathbf{0} \\
\mathbf{0} & 2 \mathbf{M}_{10}^{10} & \mathbf{0} & \mathbf{0} \\
\mathbf{0} & \mathbf{0} & \frac{1}{2} \mathbf{M}_{0 \bullet}^{0 \bullet} & \mathbf{0} \\
\mathbf{0} & \mathbf{0} & \mathbf{0} & \frac{1}{2} \mathbf{M}_{1}^{1} \mathbf{\bullet}
\end{array}\right)
\end{aligned}
$$

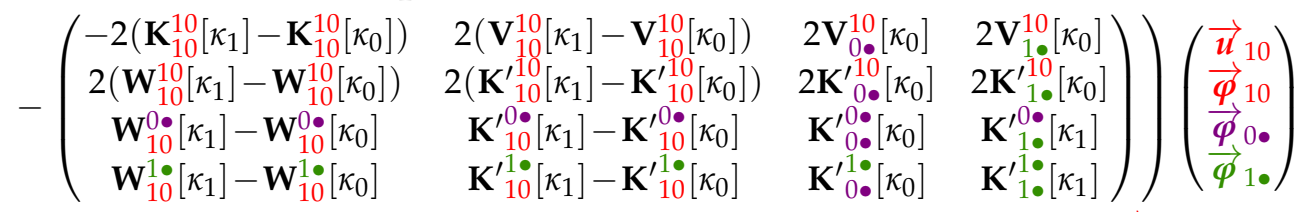

$$
\begin{aligned}
& =\left(\begin{array}{cccc}
2 \mathbf{M}_{10}^{10} & \mathbf{0} & \mathbf{0} & \mathbf{0} \\
\mathbf{0} & 2 \mathbf{M}_{10}^{10} & \mathbf{0} & \mathbf{0} \\
\mathbf{0} & \mathbf{0} & \mathbf{M}_{0 \bullet}^{0 \bullet} & \mathbf{0} \\
\mathbf{0} & \mathbf{0} & \mathbf{0} & \mathbf{M}_{1 \bullet}^{1 \bullet}
\end{array}\right)\left(\begin{array}{l}
\underset{\gamma_{D}^{1} U_{\text {inc } 10}}{\stackrel{\gamma_{N}^{1} U_{\text {inc } 10}}{\longrightarrow}} \\
\underset{\gamma_{N}^{0} U_{\text {inc } 0} \bullet}{\stackrel{\gamma_{N}^{1} U_{\text {inc } 1}}{\longrightarrow}}
\end{array}\right),
\end{aligned}
$$

with vector of unknowns $\overrightarrow{\mathfrak{u}}=\left(\overrightarrow{\boldsymbol{u}}_{10}, \overrightarrow{\boldsymbol{\varphi}}_{10}, \overrightarrow{\boldsymbol{\varphi}}_{0 \bullet}, \overrightarrow{\boldsymbol{\varphi}}_{1 \bullet}\right)^{\top} \in \mathbb{C}^{2 M_{10}+M_{0}+M_{1} \bullet}$. The vector $\overrightarrow{\mathfrak{u}}_{\text {inc }}:=\left(\overrightarrow{\gamma_{D}^{1} U_{\text {inc10 }}}, \overrightarrow{\gamma_{N}^{1} U_{\text {inc10 }}}, \overrightarrow{\gamma_{N}^{0} U_{\text {inc } 0 \bullet},}, \overrightarrow{\gamma_{N}^{1} U_{\text {inc1 }}}\right)^{\top}$ collects the coefficient vectors of the interpolant of $\left.\left.\left.\left(\gamma_{D}^{1} U_{\text {inc }}\right)\right|_{\Gamma_{10^{\prime}}}\left(\gamma_{N}^{1} U_{\text {inc }}\right)\right|_{\Gamma_{10^{\prime}}}\left(\gamma_{N}^{0} U_{\text {inc }}\right)\right|_{\Gamma_{0} \text { • }}$ and $\left.\left(\gamma_{N}^{1} U_{\text {inc }}\right)\right|_{\Gamma_{1} \mathbf{o}^{\prime}}$, respectively, see (4.2). The coefficient vector $\overrightarrow{\mathfrak{u}}_{\text {inc }}$ can be associated with an element in the finite dimensional space $V_{M} \subset \mathcal{L}_{\bullet}^{2}(\Sigma)$, see (4.4). A key observation is that

no hypersingular boundary integral operators are encountered in the interfaceoriented assembly of Galerkin matrices for (4.6),

which is another manifestation of the insight from Lemma 2.4.

The linear system of equations 4.9, arising from Galerkin discretization in the setting of Fig. 3, is rather typical. For other geometries the structure is exactly the same: when both spaces, i.e. test and trial space, are associated with a transmission interface, then matrices of the form

$$
2\left(\begin{array}{cc}
\mathbf{M}_{10}^{10} & \mathbf{0} \\
\mathbf{0} & \mathbf{M}_{10}^{10}
\end{array}\right)-\mathbf{C}_{10}^{10}
$$

occur. The mass term is dropped in the case when the test (superscript indices) and trial transmission interfaces (subscript indices) do not coincide. If we consider a block where both spaces are associated with Dirichlet interfaces, then the matrices are of the form $\mathbf{M}_{1}^{1} \bullet-\mathbf{C}_{1}^{1} \bullet$ in the case of a self-coupling and of the form $\mathbf{C}_{1}^{0 \bullet}$ in all other cases where the Dirichlet test (superscript indices) and Dirichlet trial interfaces (subscript indices) do not coincide. The coupling blocks have the form $\mathbf{C}_{0 \bullet}^{10}$, if the test space (superscript indices) is associated with a Dirichlet interface and the trial space (subscript indices) with a transmission interface, vice versa for $\mathbf{C}_{10}^{0 \bullet}$. 


\subsection{Formal Galerkin discretization for CFIE formulation 3.2}

As in the previous subsection, we consider the geometry depicted in Fig. 3. Let $G$ be the matrix on the left hand side of the linear system of equations 4.9 . We observe that the Galerkin matrix $G_{\text {CFIE }}$ arising from Formulation 3.2 is a modification of the system matrix $G$ of (4.9):

$$
G_{\mathrm{CFIE}}:=G-\mathrm{i} \eta\left(\begin{array}{cccc}
\mathbf{0} & \mathbf{0} & \mathbf{0} & \mathbf{0} \\
\mathbf{0} & \mathbf{0} & \mathbf{0} & \mathbf{0} \\
-\left(\mathbf{K}_{10}^{0 \bullet}\left[\kappa_{1}\right]-\mathbf{K}_{10}^{0 \bullet}\left[\kappa_{0}\right]\right) & \mathbf{V}_{10}^{0 \bullet}\left[\kappa_{1}\right]-\mathbf{V}_{10}^{0 \bullet}\left[\kappa_{0}\right] & \mathbf{V}_{0 \bullet}^{\mathbf{0}} \cdot\left[\kappa_{0}\right] & \mathbf{V}_{1:}^{0 \bullet}\left[\kappa_{1}\right] \\
-\left(\mathbf{K}_{10}^{1 \bullet}\left[\kappa_{1}\right]-\mathbf{K}_{10}^{1 \bullet}\left[\kappa_{0}\right]\right) & \mathbf{V}_{10}^{1 \bullet}\left[\kappa_{1}\right]-\mathbf{V}_{10}^{1 \bullet}\left[\kappa_{0}\right] & \mathbf{V}_{0 \bullet}^{1 \bullet}\left[\kappa_{0}\right] & \mathbf{V}_{1 \bullet}^{1 \bullet}\left[\kappa_{1}\right]
\end{array}\right) .
$$

For the right hand side vector we obtain

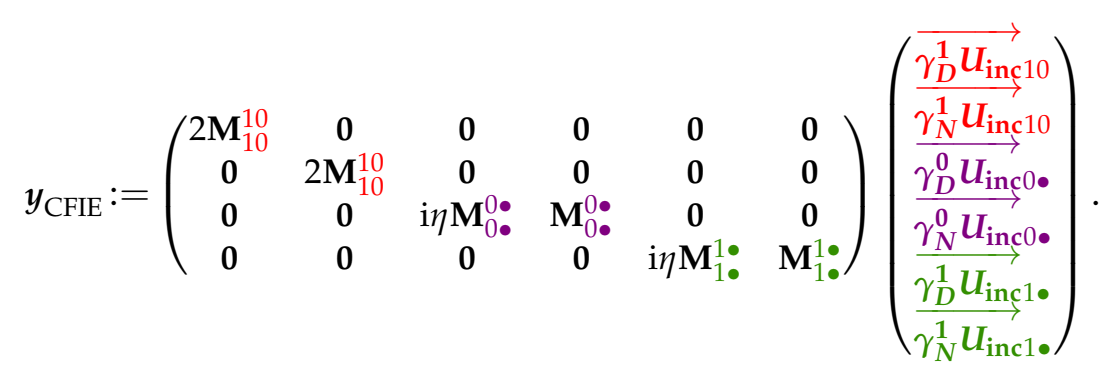

It is important to notice that in the case of CFIE, the right hand side vector also contains information about the Dirichlet data of the incident field $U_{\text {inc }}$ associated with the boundary of the impenetrable subdomain $\partial \Omega_{\text {• }}$ Namely, $\left(\overrightarrow{\gamma_{D}^{1} U_{\text {inc } 10}}, \overrightarrow{\gamma_{N}^{1} U_{\text {inc } 10}}, \overrightarrow{\gamma_{D}^{0} U_{\text {inc } 0 \bullet}}, \overrightarrow{\gamma_{N}^{0} U_{\text {inc } 0}}, \overrightarrow{\gamma_{D}^{1} U_{\text {inc } 1 \bullet},}, \overrightarrow{\gamma_{N}^{1} U_{\text {inc } 1}}\right)^{\top}$ collects the coefficient vectors of the interpolant of $\left.\left.\left.\left.\left(\gamma_{D}^{1} U_{\text {inc }}\right)\right|_{\Gamma_{10}{ }^{\prime}}\left(\gamma_{N}^{1} U_{\text {inc }}\right)\right|_{\Gamma_{10}{ }^{\prime}}\left(\gamma_{D}^{0} U_{\text {inc }}\right)\right|_{\Gamma_{0} \bullet^{\prime}} \quad\left(\gamma_{N}^{0} U_{\text {inc }}\right)\right|_{\Gamma_{0} \bullet^{\prime}}$ $\left.\left(\gamma_{D}^{1} U_{\text {inc }}\right)\right|_{\Gamma_{1} \bullet}$ and $\left.\left(\gamma_{N}^{1} U_{\text {inc }}\right)\right|_{\Gamma_{1} \bullet^{\prime}}$ respectively, see (4.2).

This yields the following linear system of equations generated by the Galerkin BEM discretization of CFIE Formulation 3.2:

$$
G_{\text {CFIE }}\left(\vec{u}_{10}, \vec{\varphi}_{10}, \vec{\varphi}_{0 \bullet}, \vec{\varphi}_{1 \bullet}\right)^{\top}=y_{\mathrm{CFIE}^{\prime}}
$$

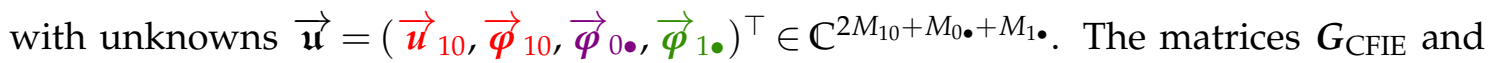
$y_{\text {CFIE }}$ are given in (4.10) and (4.11), respectively.

\subsection{Convergence and conditioning}

Since we can show only that the operator on the left hand side of Formulation 2.3 is a Fredholm operator of index zero, see also Remark 2.1, we cannot assert quasi-optimality of Galerkin solutions of Formulations 2.3 and 3.2. Yet, bolstered by what we have universally observed in numerical tests, we may assume stability of our Galerkin discretization. 
Assumption 4.1 (Discrete Inf-Sup Conditions). We assume that Formulation 2.3, discretized by means of low-order piecewise polynomial boundary element spaces on shape-regular sequences of meshes, satisfies an asymptotic uniform discrete inf-sup condition (see [35, Theorem 4.2.7]).

Then, we conclude by [35, Theorem 4.2.7] well-posedness, asymptotic stability, and quasi-optimality of the Galerkin discretization. Assume a shape-regular and quasiuniform sequence $\left\{\mathcal{T}_{\ell}\right\}_{\ell \in \mathbb{N}}$ of skeleton meshes with

$$
h_{\ell}:=\max \left\{\operatorname{diam}(\tau), \tau \in \mathcal{T}_{\ell}\right\} \rightarrow 0 \text { as } \ell \rightarrow \infty,
$$

see [37, Section 10.1] $(d=2)$ or [35, Section 4.1.2] $(d=3)$ for details. Under these circumstances, quasi-optimality implies $\mathcal{O}\left(h_{\ell}\right)$ algebraic convergence of the discretization error in the $\mathcal{L}_{\bullet}^{2}(\Sigma)$-norm as a consequence of best approximation error estimates from $[37$, Section 10.1] or [35, Section 4.1.2].

The condition numbers of the Galerkin matrices crucially depend on the choice of basis functions. In particular, for $V_{M_{\ell}}=V_{\mathcal{T}_{\ell}, 0}$, see (4.5), we choose the canonical basis of characteristic functions of mesh cells. This basis is perfectly $L^{2}$-stable, since, after suitable scaling, it is even $L^{2}$-orthonormal. Thus, by the continuity of $\mathbf{M}_{\Sigma}: \mathcal{M} \mathcal{L}^{2}(\Sigma) \rightarrow \mathcal{M} \mathcal{L}^{2}(\Sigma)$ and appealing to Assumption 4.1, we can conclude that the Euclidean condition numbers of the Galerkin matrices $\mathbf{G}_{\ell} \in \mathbb{C}^{M_{\ell}, M_{\ell}}$ arising from Formulation 3.2 are bounded independently of $h_{\ell}$.

\subsection{Post-processing}

Assume that we perform a Galerkin discretization of Formulation 2.3 based on the boundary element space $V_{M_{\ell}}=V_{\mathcal{T}_{\ell}, 0}$ of piecewise constant functions on quasi-uniform sequences of triangular or quadrilateral meshes $\left\{\mathcal{T}_{\ell}\right\}_{\ell \in \mathbb{N}}$ obtained by regular refinement. In this case, we observe a gain in accuracy if we perform a simple and cheap postprocessing, which relies on the boundary element space $\mathcal{S}_{\mathcal{T}_{\ell}}^{1,0}(\Sigma)$ of continuous, piecewise linear functions on $\mathcal{T}_{\ell}$, see [35, Def. 4.1.36] $(d=3)$, [37, Sect. 10.2] $(d=2)$.

The post-processing we recommend boils down to computing the $L^{2}$-projection of the Dirichlet data of the discrete solution onto the continuous piecewise linear boundary element space $\mathcal{S}_{\mathcal{T}_{\ell}}^{1,0}(\Sigma)$. Algorithmically, the projection entails the inversion of a sparse mass matrix for the space $\mathcal{S}_{\mathcal{T}_{f}}^{1,0}(\Sigma)$. When using modern direct sparse elimination solvers the extra computational effort is negligible compared to the cost of handling the boundary element matrices.

\section{$5 \quad$ Numerical experiments}

This section is dedicated to numerical experiments testing the performance of our new second-kind Formulations 2.3 and 3.2 compared to the classical first-kind formulation 


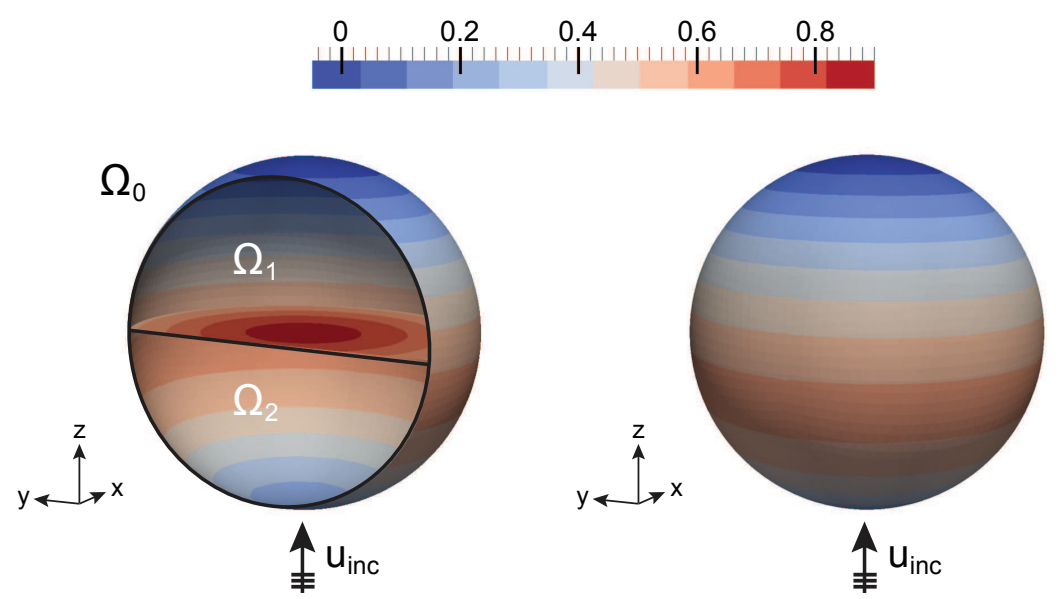

Figure 4: Geometry of the scatterer in Experiment I. Shown is the real part of the total field $U$.

from [38] for $d=3 .^{+t}$ While for the second-kind approach, we use piecewise constant boundary element test and trial spaces (see (4.5)), for the first-kind approach we need to use $\mathcal{S} \mathcal{T}(\Sigma)$-conforming boundary element spaces. We take continuous piecewise linear boundary elements $\mathcal{S}_{\mathcal{T}_{\ell}}^{1,0}(\Sigma)$ to discretize Dirichlet data and $\mathcal{S}_{\mathcal{T}_{\ell}}^{0,-1}(\Sigma)$ for Neumann data. The meshes $\left\{\mathcal{T}_{\ell}\right\}_{\ell \in \mathbb{N}}$ we used for the experiments consisted of flat triangular cells.

We are going to discuss three different scattering problems based on the geometries depicted in Figs. 4, 5 and 6, respectively.

\subsection{Experiment I: Transmission problem}

The first experiment solves the scattering problem (1.2a), (1.2b) at the composite scatterer shown in Fig. 4 for the incident plane wave $U_{\text {inc }}(x)=\exp \left(\mathrm{i} \kappa_{0} \boldsymbol{d} \cdot \boldsymbol{x}\right)$, with direction of propagation $\boldsymbol{d}:=(0,0,1)^{\top}$. The scatterer consists of two different materials. The first part of the scattering obstacle is given by the ball $\mathcal{B}_{0.4}(\mathbf{0})$ of radius $r=0.4$, centered in 0 and halved by the plane $\mathcal{E}:=\left\{x=(x, y, z)^{\top} \in \mathbb{R}^{3}: z=0\right\}$. The upper half of the ball in Fig. 4, i.e. $\Omega_{1}:=\mathcal{B}_{0.4}(\mathbf{0}) \cap\left\{x=(x, y, z)^{\top} \in \mathbb{R}^{3}: z>0\right\}$ is filled with a penetrable medium characterized by the wave number $\kappa_{1}=5$. The lower half of the ball, denoted by $\Omega_{2}:=\mathcal{B}_{0.4}(\mathbf{0}) \cap\left\{\boldsymbol{x}=(x, y, z)^{\top} \in \mathbb{R}^{3}: z<0\right\}$ is also penetrable and with wave number $\kappa_{2}=1$. The exterior domain $\Omega_{0}:=\mathbb{R}^{3} \backslash \overline{\mathcal{B}_{0.4}(\mathbf{0})}$ has wave number $\kappa_{0}=2$.

\footnotetext{
${ }^{++}$The implementation was done based on the C++ BEM library "Boundary Element Template Library 2" (BETL2), developed by L. Kielhorn [25].

怙The meshes were generated with GMSH [18] and for visualization of the computed data (see Figs. 4, 5, 6) we used ParaView [1]. All other plots were generated with MATLAB.
} 


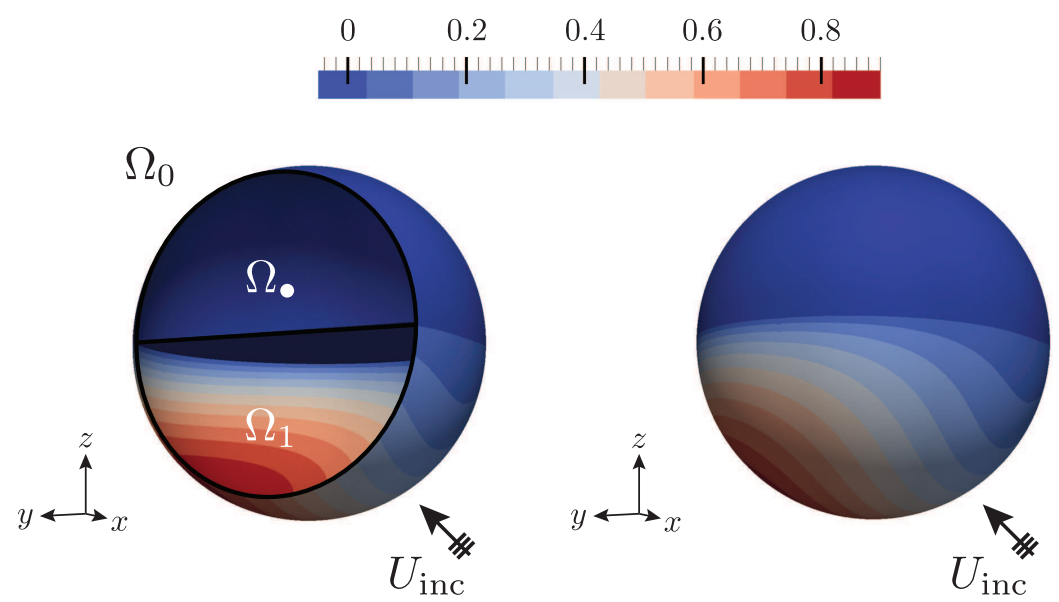

Figure 5: Geometry of the scatterer in Experiment II. Shown is the real part of the total field $U$.

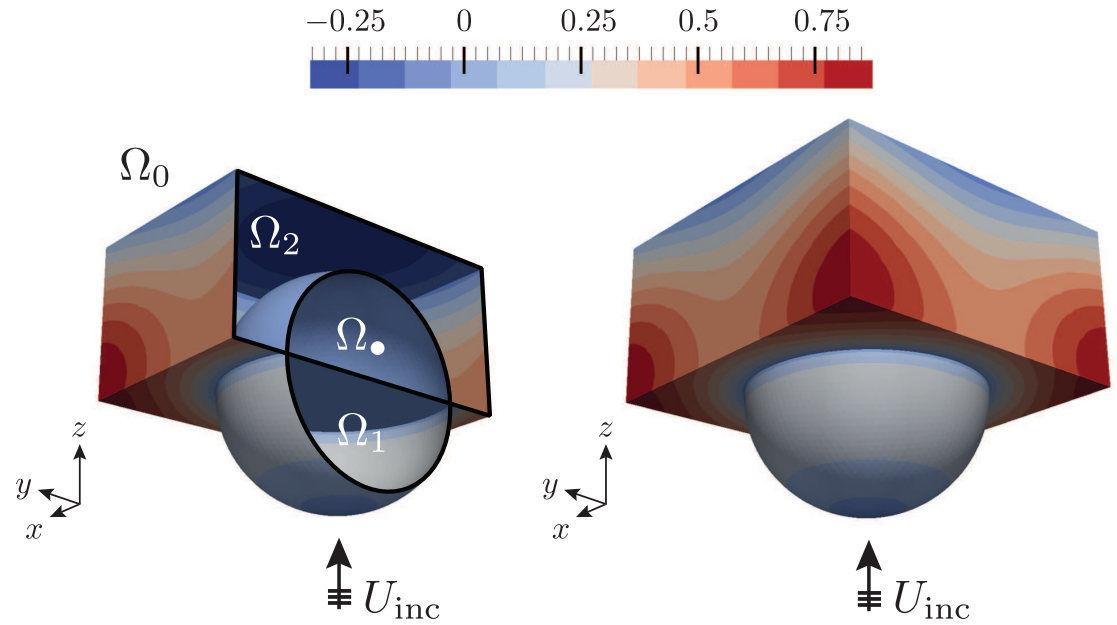

Figure 6: Geometry of the scatterer in Experiment III. Shown is the real part of the total field $U$.

\subsection{Experiment II: Transmission problem with impenetrable part}

We solve the acoustic scattering problem with incident plane wave toward direction $\mathbf{d}=$ $\frac{1}{\sqrt{2}}(0,1,1)^{\top}$ hitting a ball-shaped scattering object $\mathcal{B}_{0.5}(\mathbf{0})$ of radius $r=0.5$, centered at the origin. A picture of the geometry is given in Fig. 5. We impose homogeneous Dirichlet boundary conditions on $\partial \Omega_{\bullet}$, where $\Omega_{\bullet}:=\mathcal{B}_{0.5}(\mathbf{0}) \cap\left\{(x, y, z)^{\top} \in \mathbb{R}^{3}: z>0\right\}$ The other half of the ball, $\Omega_{1}:=\mathcal{B}_{0.5}(\mathbf{0}) \cap\left\{(x, y, z)^{\top} \in \mathbb{R}^{3}: z<0\right\}$ is penetrable and characterized by $\kappa_{1}=4$. The exterior domain $\Omega_{0}:=\mathbb{R}^{3} \backslash \overline{\mathcal{B}_{0.5}(\mathbf{0})}$ has the wave number $\kappa_{0}=2$. 


\subsection{Experiment III: Complex transmission problem with impenetrable part}

The incident plane wave in Experiment III is incoming with direction $\mathbf{d}=(0,0,1)^{\top}$. The scatterer, which is depicted in Fig. 6, consists of three different materials. The first part of the scattering obstacle is given by the ball $\mathcal{B}_{0.5}(\mathbf{0})$, with $\Omega_{\bullet}:=\mathcal{B}_{0.5}(\mathbf{0}) \cap\left\{(x, y, z)^{\top} \in \mathbb{R}^{3}: z>\right.$ $0\}$, and $\Omega_{1}:=\mathcal{B}_{0.5}(\mathbf{0}) \cap\left\{(x, y, z)^{\top} \in \mathbb{R}^{3}: z<0\right\}$. $\Omega_{\bullet}$ is impenetrable and characterized by homogeneous Dirichlet boundary conditions on $\partial \Omega_{\bullet}$, while $\Omega_{1}$ is a penetrable medium characterized by $\kappa_{1}=1$. In addition, we consider another medium given through $\kappa_{2}=4$ in $\Omega_{2}:=\mathcal{Q} \backslash \overline{\mathcal{B}_{0.5}(\mathbf{0})}$ where $\mathcal{Q}:=\left\{(x, y, z)^{\top} \in \mathbb{R}^{3}: 0.7<x<-0.7,0.7<y<-0.7,0.7<z<0\right\}$. The exterior domain $\Omega_{0}:=\mathbb{R}^{3} \backslash \overline{\left(\mathcal{B}_{0.5}(\mathbf{0}) \cup \mathcal{Q}\right)}$ is penetrable with wave number $\kappa_{0}=2$.

\subsection{Convergence and post-processing}

We consider a sequence of nested meshes $\left\{\mathcal{T}_{\ell}\right\}_{\ell=1}^{H}$ with $H=6$. The corresponding number of elements are $\{40,160,640,2560,10240,40960\}$ for Experiment I, $\{44,176,704,2816,11264,45056\}$ for Experiment II, and $\{140,560$, $2240,8960,35840,143360\}$ for Experiment III. They are created by uniform refinement and consist of flat, uniformly shape regular triangular elements. The local mesh width is calculated as the maximal distance of the center of mass to all points lying inside of the element. The global mesh width $h_{\ell}$ is given by the maximum over all local mesh widths. In Fig. 7, we show the convergence of the discretization error in $L^{2}(\Sigma)$ and $H^{-\frac{1}{2}}(\Sigma)$-norm, respectively, with respect $h_{\ell}$. As a reference solution we use the discrete solution calculated with the second-kind formulation on the finest grid $\mathcal{T}_{H}$. The convergence rates are as expected.

The term proj. Dirichlet second-kind denotes a post-processed version of the Dirichlet data of the second-kind solution, obtained by projecting the computed data onto the space of continuous piecewise linear boundary elements $\mathcal{S}_{\mathcal{T}}^{1,0}(\Sigma)$ in the $L^{2}$-sense (see Subsection 4.5).

The convergence plot in Fig. 7 shows that the application of this cheap postprocessing technique improves the convergence rate and we observe results that are as good as the results of the classical first-kind approach.

\subsection{Conditioning of Galerkin matrices}

In Fig. 8 the Euclidean condition number of the Galerkin matrices is plotted with respect to the inverse of the mesh width $h_{\ell}$ of the discretization. As expected from the $L^{2}$-stability of the characteristic basis used for our second-kind Galerkin approximation, see Section 4.1, we observe condition numbers for the Galerkin matrix that are almost independent of the mesh size, while the condition numbers of the Galerkin matrices of the first-kind approach blow up like $\mathcal{O}\left(h_{\ell}{ }^{-2}\right)$ (see Fig. 8, compare with [35, Section 4.5 \& Cor. 6.4.14]). This behavior is directly reflected by the iteration count for the iterative solver GMRES, as can be seen in Fig. 9. 

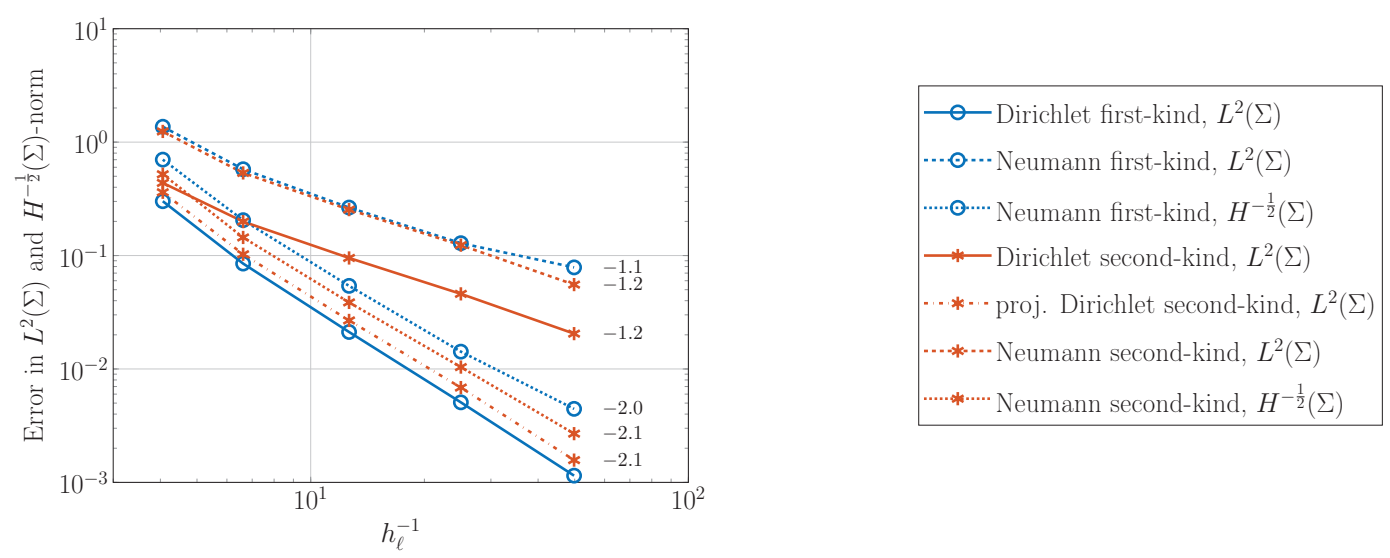

(a) Experiment I

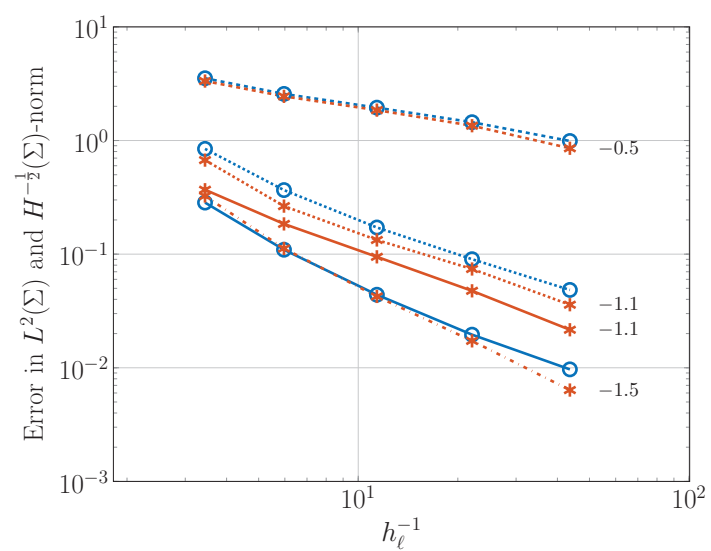

(b) Experiment II

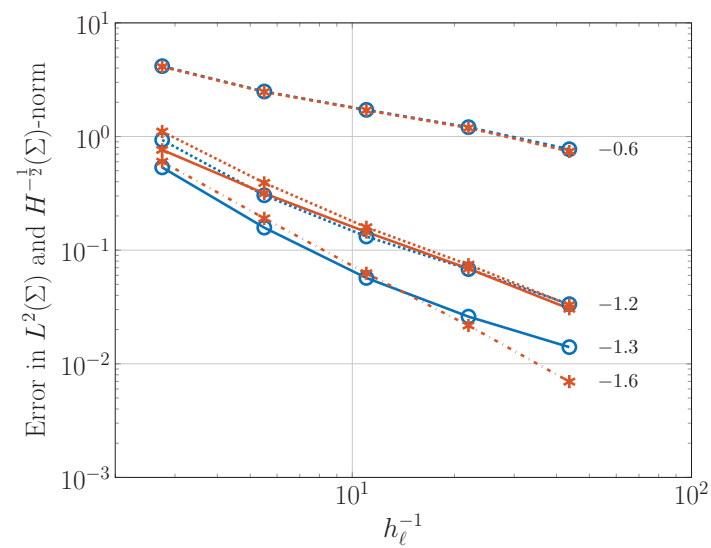

(c) Experiment III

Figure 7: Convergence of the error of (diagonally rescaled) first- and second-kind Galerkin discretization in $L^{2}$ and $H^{-\frac{1}{2}}$-norm for a sequence of meshes.

\subsection{Spurious resonances due to impenetrable objects}

We tested for spurious resonances by monitoring the condition numbers of the Galerkin matrices while varying wave numbers. In the case of a single uniform ball-shaped impenetrable scatterer $\Omega_{\bullet}:=\mathcal{B}_{r}(\mathbf{0})$, we can explicitly compute the wave numbers $\kappa_{0}$ for which we will observe a spurious resonance (see also Section 3 ). They correspond to the roots of derivatives of spherical Bessel functions and the roots of the spherical Bessel functions, respectively, scaled by $\frac{1}{r}$.

We take $r=0.5$ and consider the range of wave numbers between 8.8 and 9.2. This range includes 8.986, which corresponds to $\frac{1}{r} x$, where $x=4.493$ is the first root of the spherical Bessel function $j_{1}$ and the second root of the derivative of the spherical Bessel function $j_{0}$. Fig. 5.6 shows the Euclidean condition numbers of the Galerkin matrices. 


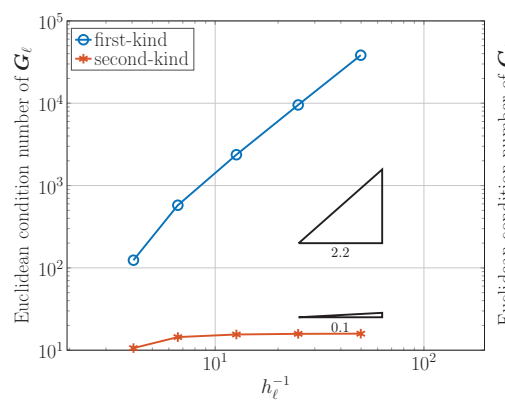

(a) Experiment I

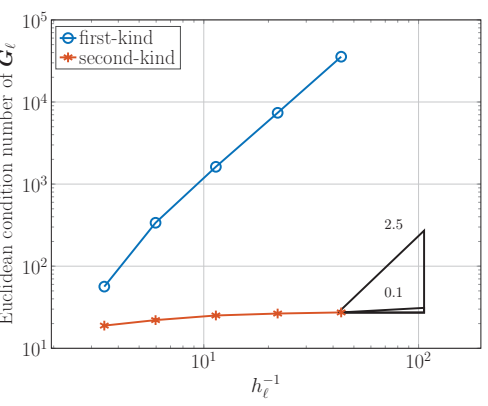

(b) Experiment II

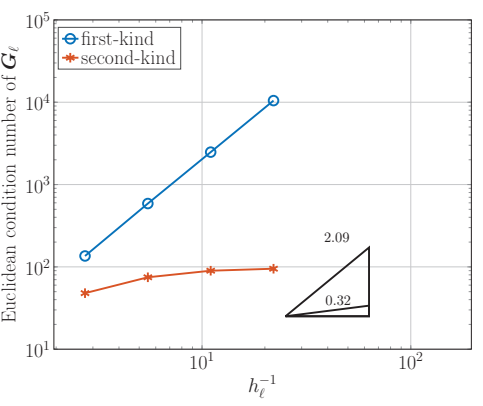

(c) Experiment III

Figure 8: Condition numbers of (diagonally rescaled) first- and second-kind Galerkin matrices for a sequence of meshes.

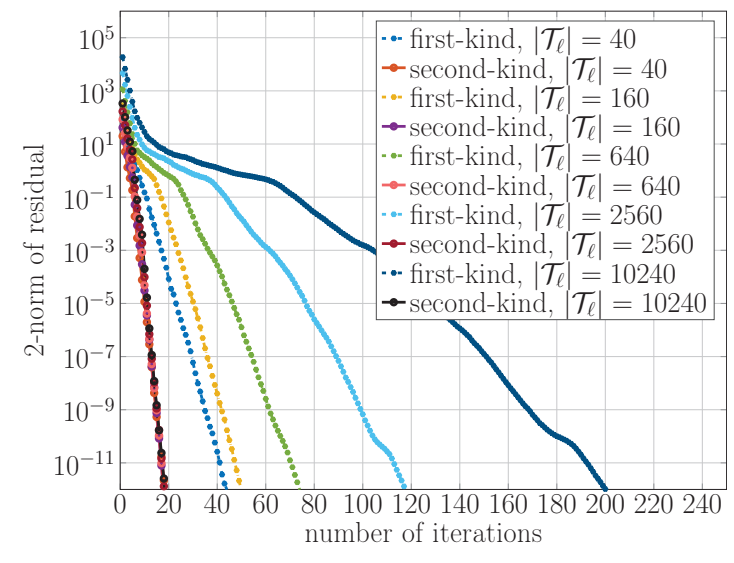

(a) Experiment I

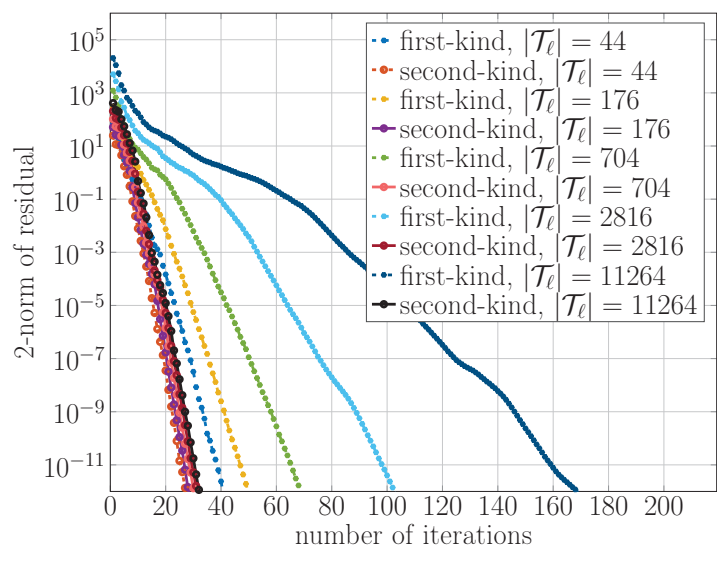

(b) Experiment II

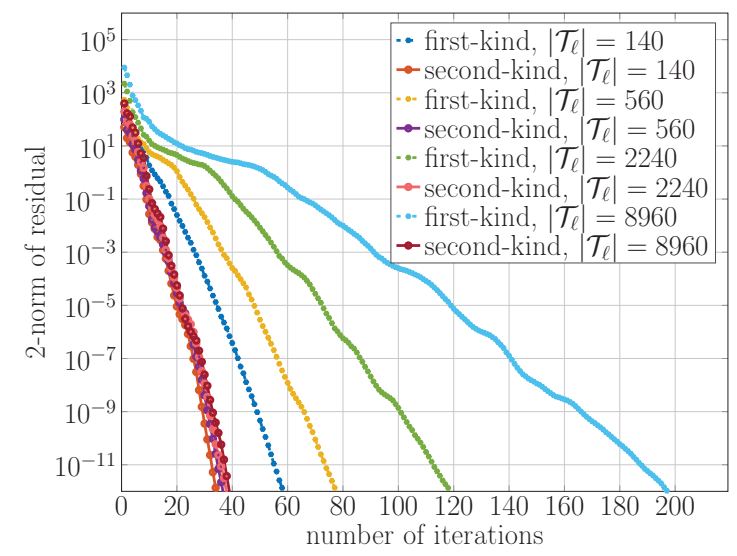

(c) Experiment III

Figure 9: Convergence of GMRES applied to (diagonally rescaled) first- and second-kind Galerkin system. 


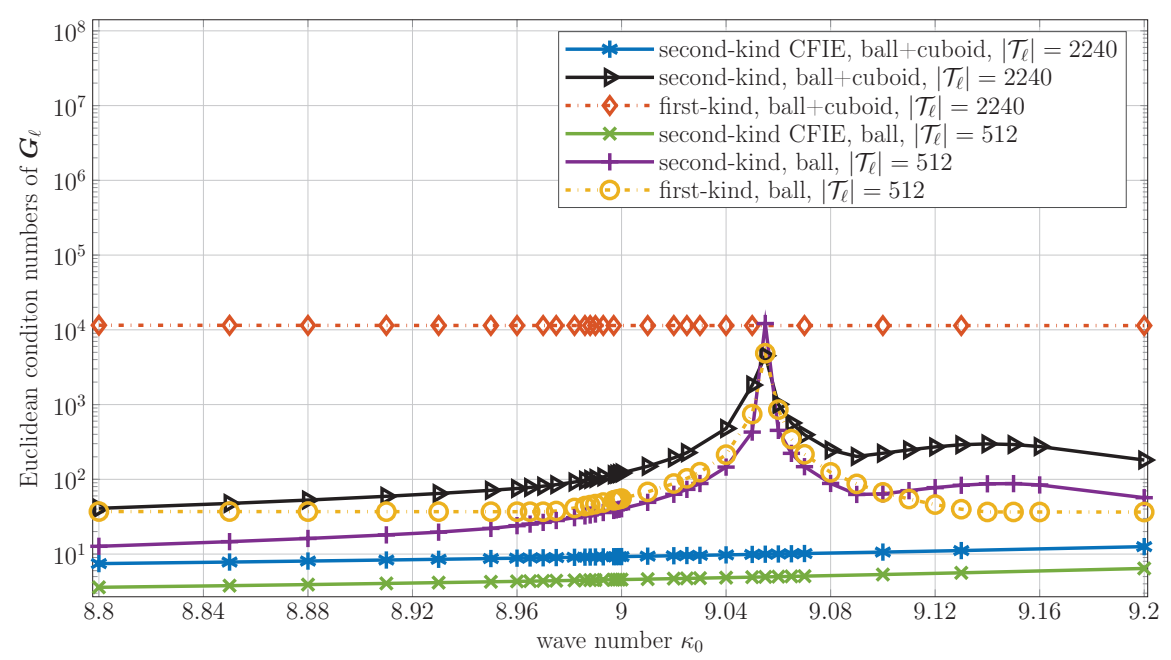

Figure 10: Spurious resonances due to impenetrable objects: condition numbers of the first- and second-kind Galerkin matrix for various wave numbers. In the case of the geometry ball, the scatterer consists of the ball $\mathcal{B}_{0.5(0)}$ and is impenetrable, while in the case of the geometry ball+cuboid, which refers to the geometry of Experiment III, we set $\kappa_{0}=\kappa_{2}$ and assume that $\Omega_{1}$ is impenetrable as well.

Since we are computing the Galerkin matrices only on relatively coarse comprising 512 and 2240 elements, we observe a shift of the resonance due to approximation errors. Obviously, the spurious resonances disappear when using a CFIE approach.

In addition to the simple ball-shaped scatterer $\mathcal{B}_{0.5}(\mathbf{0})$, we also consider the geometry shown in Fig. 6, taking the cuboid as pseudo-interface, i.e. setting $\kappa_{2}=\kappa_{0}$, and defining $\Omega_{1}$ to be impenetrable, too. In this setting, in accordance with the theory of [8], we get rid of the spurious resonances in the case of the first-kind formulation. In the case of the second-kind formulation, spurious resonances persist. However, the condition numbers for the first-kind formulation with pseudo-interface increase drastically such that the use of pseudo-interfaces for first-kind formulations does not pay off.

\section{Conclusion}

In this paper, we have extended the second-kind Galerkin boundary element method proposed in [12] to partly impenetrable composite scatterers. We also devised a combinedfield approach meant to overcome spurious resonances due to the impenetrable parts. Numerical experiments show that the new method is superior to the widely used classical first-kind approach [38]: it produces Galerkin matrices that are intrinsically wellconditioned and allow fast iterative solution without preconditioning. Its implementation can rely on standard algorithms for singular integration, and its Galerkin solutions are as accurate as those obtained with the first-kind approach when applying a cheap post-processing technique. Throughout our tests, the new formulation has demonstrated to be perfectly stable, though this property has eluded a rigorous proof up to now. 


\section{Acknowledgments}

The authors would like to thank L. Kielhorn for his great support during the development of the code for the first- and second-kind formulation in BETL2 [25]. The work of E. Spindler was partially supported by SNF under grant 20021_137873/1. X. Claeys received support from the ANR Research Grant ANR-15-CE23-0017-01.

\section{References}

[1] U. Ayachit. The ParaView Guide: A Parallel Visualization Application. Kitware, 2015.

[2] J. M. Ball, Y. Capdeboscq, and B. T. Xiao. On uniqueness for time harmonic anisotropic Maxwell's equations with piecewise regular coefficients. Math. Models Meth. Appl. Sci., 2012.

[3] A. Buffa and R. Hiptmair. Regularized Combined Field Integral Equations. Numerische Mathematik, Springer, 100(1):1-19, 2005

[4] A. J. Burton and G. F. Miller. The application of integral equation methods to the numerical solution of some exterior boundary-value problems. Proceedings of the Royal Society of London A: Mathematical, Physical and Engineering Sciences, 323(1553):201-210, 1971.

[5] Y. Chang and R. Harrington. A surface formulation for characteristic modes of material bodies. IEEE Transactions on Antennas and Propagation, 25(6):789-795, 1977.

[6] X. Claeys. A single trace integral formulation of the second kind for acoustic scattering. Tech. Rep. 2011-14, Seminar for Applied Mathematics, ETH Zürich, 2011.

[7] X. Claeys and R. Hiptmair. Multi-trace boundary integral formulation for acoustic scattering by composite structures. Communications on Pure and Applied Mathematics, 66: 11631201, 2013.

[8] X. Claeys and R. Hiptmair. Integral Equations for Acoustic Scattering by Partially Impenetrable Composite Objects. Integral Equations Operator Theory, 81(2): 151-189, 2015.

[9] X. Claeys, R. Hiptmair, and C. Jerez-Hanckes. Multitrace boundary integral equations. Direct and Inverse Problems in Wave Propagation and Applications, Radon Series on Computational and Applied Mathematics, 14:51-100, 2013.

[10] X. Claeys, R. Hiptmair, C. Jerez-Hanckes, and S. Pintarelli. Novel Multi-Trace Boundary Integral Equations for Transmission Boundary Value Problems. Unified Transform for Boundary Value Problems: Applications and Advances, SIAM, 2015.

[11] X. Claeys, R. Hiptmair, and E. Spindler. Second-kind boundary integral equations for electromagnetic scattering at composite objects. Preprint 2016-43, SAM, ETH Zurich, Switzerland, 2016. Submitted to Computers \& Mathematics with Applications.

[12] X. Claeys, and R. Hiptmair, and E. Spindler. A second-kind Galerkin boundary element method for scattering at composite objects. BIT Numerical Mathematics, 55(1):33-57, 2015.

[13] X. Claeys, R. Hiptmair, and E. Spindler. Second kind boundary integral equation for multisubdomain diffusion problems. Adv. Comp. Math., 2017. DOI 10.1007/s10444-017-9517-0.

[14] D. Colton and R. Kress. Inverse Acoustic and Electromagnetic Scattering Theory. Vol. 93. Springer, 2012.

[15] M. Costabel and E. P. Stephan. A direct boundary equation method for transmission problems. J. Math. Anal. Appl., 106:367-413, 1985.

[16] M. Costabel and E. P. Stephan. Boundary integral equations for mixed boundary value problems in polygonal domains and Galerkin approximation. Banach Center Publications, 15(1):175-251, 1985.

[17] J. Elschner. The double layer potential operator over polyhedral domains I: Solvability in weighted Sobolev spaces. Applicable Analysis 45(1-4):117-134, 1992. 
[18] C. Geuzaine and J.-F. Remacle. Gmsh: a three-dimensional finite element mesh generator with built-in pre- and post-processing facilities. International Journal for Numerical Methods in Engineering, 79(11):1309-1331, 2009.

[19] L. Greengard and J.Y. Lee. Short note: Stable and accurate integral equation methods for scattering problems with multiple material interfaces in two dimensions. Journal of Computational Physics, 231(6):2389-2395, 2012.

[20] P. Grisvard. Singularities in boundary value problems. Recherches en mathématiques appliquées, Masson, 1992.

[21] R.F. Harrington. Boundary integral formulations for homogeneous material bodies. J. Electromagnetic Waves and Applications, 3(1):1-15, 1989.

[22] C. Hazard and M. Lenoir. On the solution of time-harmonic scattering problems for Maxwell's equations. SIAM J. Math. Anal., 27(6):1597-1630, 1996.

[23] R. Hiptmair. Operator preconditioning. Computers and Mathematics with Applications, 52:699_ 706, 2006

[24] R. Hiptmair and C. Jerez-Hanckes. Multiple traces boundary integral formulation for Helmholtz transmission problems. Adv. Appl. Math., 37:39-91, 2012.

[25] R. Hiptmair and L. Kielhorn. BETL - a generic boundary element template library. Tech. Rep. 2012-36, Seminar for Applied Mathematics, ETH Zürich, 2012.

[26] D. Jerison and C. E. Kenig. The inhomogeneous Dirichlet problem in Lipschitz domains. J Funct. Anal., 130(1):161-219, 1995.

[27] R. Kress and G. F. Roach. Transmission problems for the Helmholtz equation. Journal of Mathematical Physics, 19(6):1433-1437, 1978.

[28] W. McLean. Strongly Elliptic Systems and Boundary Integral Equations. Cambridge University Press, Cambridge, UK, 2000.

[29] A.-W. Maue. Zur Formulierung eines allgemeinen Beugungsproblems durch eine Integralgleichung. Zeitschrift für Physik, 126(7-9):601-618, 1949.

[30] C. Müller. Foundations of the mathematical theory of electromagnetic waves. Springer, 1969.

[31] Z. Peng, K.-H. Lim, and J.-F. Lee. Computations of electromagnetic wave scattering from penetrable composite targets using a surface integral equation method with multiple traces. IEEE Trans. Antennas and Propagation, 61:256-270, 2013.

[32] Z. Peng, X.-C. Wang, and J.-F. Lee. Integral equation based domain decomposition method for solving electromagnetic wave scattering from non-penetrable objects. IEEE Trans. Antennas and Propagation, 59:33283338, 2011.

[33] A. J. Poggio and E. K. Miller. Integral equation solution of three-dimensional scattering problems. Computer Techniques for Electromagnetics, chapter 4, p. 159-263, New York, 1973.

[34] V. Rokhlin. Solution of acoustic scattering problems by means of second kind integral equations. Wave Motion, 5(3):257-272, 1983.

[35] S. A. Sauter and C. Schwab. Boundary element methods. Springer, 2011.

[36] E. Spindler, Second Kind Single-Trace Boundary Integral Formulations for Scattering at Composite Objects, ETH dissertation no. 23620, ETH Zurich, Switzerland, 2016.

[37] O. Steinbach. Numerical approximation methods for elliptic boundary value problems: finite and boundary elements. Springer, 2008.

[38] T. Von Petersdorff. Boundary integral equations for mixed Dirichlet, Neumann and transmission problems. Mathematical methods in the applied sciences, 11(2):185-213, 1989.

[39] T.-K. Wu and L. L. Tsai. Scattering from arbitrarily-shaped lossy dielectric bodies of revolution. Radio Science, 12(5): 709 - 718, 1977. 